\title{
Review of research into shock control bumps
}

\author{
P. J. K. Bruce · S. P. Colliss
}

Received: 30 October 2013 / Revised: 22 April 2014 / Accepted: 8 September 2014 / Published online: 28 October 2014

(C) The Author(s) 2014. This article is published with open access at Springerlink.com

\begin{abstract}
This paper presents a review of research on shock control bumps (SCBs), a class of flow control device with potential for application to transonic wings. Beginning with a brief review of the origins of the SCB concept, the primary focus is on the more recent studies from the last decade. Results from both experimental and numerical work are considered and the synergy between these two approaches to SCB research is critically explored. It is shown that the aerodynamic performance enhancement potential of SCBs, namely their capacity for drag reduction and delaying the onset of buffet for transonic wings, has been widely demonstrated in the literature, as has the high sensitivity of SCB performance to flow conditions including shock strength and position, and post-shock adverse pressure gradient. These characteristic features of SCBs are relatively well explained in terms of the flow physics that have been observed for different bump geometries. This stems from a number of studies that have focused on the balance of viscous and inviscid flow features and also the mechanism by which finite span SCBs generate streamwise vorticity. It is concluded that our understanding of SCBs is reaching an advanced level of maturity for SCBs in simple configurations and steady flow fields. However, SCB performance in unsteady flow and on swept wings requires further investigation before the concept can be considered a viable candidate for transonic wings. These investigations should adopt a multi-disciplinary approach combining carefully designed experiments and targeted computations. Finally, two concepts for future SCB research are suggested: the adaptive SCB and SCBs in engine intakes.
\end{abstract}

Communicated by A. Hadjadj.

P. J. K. Bruce $(\varangle) \cdot$ S. P. Colliss Imperial College London, London, UK

e-mail: p.bruce@imperial.ac.uk
Keywords Transonic aerodynamics - Flow control · Shock control bump

\section{List of symbols}

\begin{tabular}{|c|c|}
\hline$M_{\infty}$ & Freestream Mach number \\
\hline$\delta_{0}$ & Incoming boundary layer thickness \\
\hline$\delta_{i}^{*} \equiv \int_{0}^{\delta}\left(1-\frac{u}{u_{e}}\right) \mathrm{d} y$ & $\begin{array}{l}\text { Kinematic boundary layer } \\
\text { displacement thickness }\end{array}$ \\
\hline$\theta_{i} \equiv \int_{0}^{\delta} \frac{u}{u_{e}}\left(1-\frac{u}{u_{e}}\right) \mathrm{d} y$ & $\begin{array}{l}\text { Kinematic boundary layer } \\
\text { momentum thickness }\end{array}$ \\
\hline$H_{i} \equiv \delta_{i}^{*} / \theta_{i}$ & Boundary layer shape factor \\
\hline
\end{tabular}

\section{Scope}

Shock control bumps (SCBs) are a relatively new class of flow control device that offer a number of potential performance benefits for (transonic or supersonic) applications where shock waves are present. They have most commonly been considered as a means for improving the performance of transonic wings, through the manipulation of the airflow over the upper wing surface where a near-normal shock wave exists. As with all flow control devices, research has shown that SCBs invariably offer a compromise between a number of beneficial and detrimental effects when installed on a transonic aerofoil or wing. In this context, it is informative (although perhaps not entirely rigorous) to make the statement that the vast majority of studies have assessed the performance potential of SCBs in terms of one or both of the following two criteria:

- Their impact on drag at flow conditions which are optimal for the SCB but not necessarily the same as the original aerofoil's optimal design point. 
- Their impact on the off-design performance of an aerofoil or wing, in terms of phenomena such as buffet.

The subtle details of how different geometry SCBs fulfil these two (often conflicting) criteria is essentially the main topic of this review article. Broadly speaking, a glance through the relevant literature from the past two decades reveals that studies of SCBs fall into one of the two categories: (1) Investigations that consist of parametric studies and/or optimisation; and (2) Investigations that probe the detailed flow physics produced by SCBs in controlled environments. In this review article, we will draw together the results from studies of both types. It is logical to first consider the detailed flow physics produced by SCBs before attempting to assess their performance potential. We will consider the potential of SCBs to improve existing aerofoils and also their potential as a means for facilitating a new generation of unconventional geometry transonic aerofoils (specifically laminar-flow aerofoils).

This review article proceeds as follows: Firstly, the concept of a SCB is introduced at a basic level in Sect. 2 in terms of its defining geometry, the flow structure(s) it produces and basic performance trends. This is followed in Sect. 3 by a more in-depth analysis of several important flow features associated with SCBs that have been identified in recent studies. The impact of SCB geometry and local and global flow conditions on these flow features is also reported. SCB performance is considered briefly in Sect. 4, including a comparison of SCBs with other flow control techniques. Finally, directions for future research and development are proposed in Sect. 5.

\section{Introduction to $\mathrm{SCBS}$}

\subsection{Historical background}

Early days. The origins of the SCB can be traced back to the late 1970s, where researchers investigating early transonic aerofoil designs considered designing a humped transonic aerofoil to mitigate problems caused by high-shock strength and maximise the aerofoil's drag-divergence Mach number [34]. The potential of these humped supercritical aerofoils to improve off-design performance (i.e. delay buffet and increase drag-divergence Mach number) was confirmed by experiments in the 1980s [35], although the results also showed that there was significant room for further improvement. In 1992, Ashill et al. [2] were the first to consider $\mathrm{SCBs}$ as a method for wave drag reduction on laminarflow aerofoils. Their results were promising and stimulated a significant research effort in the following decade, with major projects in Europe (Euroshock II [33]) and in the US
(NASA's Aircraft Morphing project [20]) with significant elements dedicated to exploring the potential of SCBs.

2000- These projects and other early studies effectively confirmed the performance benefits that could be achieved with SCBs. In the last ten-or-so years, there has been a subtle shift in the direction of SCB research, with a greater emphasis on refining our knowledge of the detailed flow physics that SCBs produce in an effort to better understand how to optimise their performance and overcome the challenges facing their integration into current and future aircraft. Progress in computational studies of SCBs has been significant with many high quality results appearing very recently that have contributed to our understanding. On the experimental side, there has been a move away from relatively large scalemodel validation-type tests (where the objective is invariably to obtain values of $L / D$ ) towards more fundamental tests in carefully controlled environments (where high resolution local measurements are sought). Collaborative experimental/computational research into SCBs has been a major focus of the recent NextWing project, which has involved partners from the University of Cambridge and IAG Stuttgart as part of the EU Clean Sky research project.

\subsection{Geometry}

As the name suggests, conventional SCBs consist of a physical bump placed on an aerodynamic surface where a shock wave is known to occur. Although some researchers have considered using SCBs in supersonic flows with shock waves (such as engine intakes, see, for example $[15,16]$ ), by far the most common application considered is the upper surface of a transonic wing. In this application, a SCB can have a beneficial smearing effect on the structure of the near-normal shock wave close to the wing surface. Specifically, SCBs split the shock into a number of weaker (oblique) shocks or compression waves that decelerate the flow more gradually (and hence more isentropically) than the (single) uncontrolled shock wave, thus incurring a reduced stagnation pressure loss and lower drag. This principle is illustrated in Fig. 1.

A typical SCB consists of a ramp upstream of the nominal shock location, followed by a short crest region and then a tail. The ramp generates an oblique shock (or multiple oblique compression waves) ahead of the main shock wave which deflects the incoming supersonic flow away from the surface. Around the crest region, the flow is decelerated to subsonic velocities by a near-normal shock wave, before the tail brings the post-shock flow back to the aerofoil surface. Figure 2 illustrates the shock structure produced by two different geometry SCBs: one with a smoothly contoured shape and another with a more wedge-like design, as reported by Birkemeyer et al. [4] and Ogawa et al. [26]. 

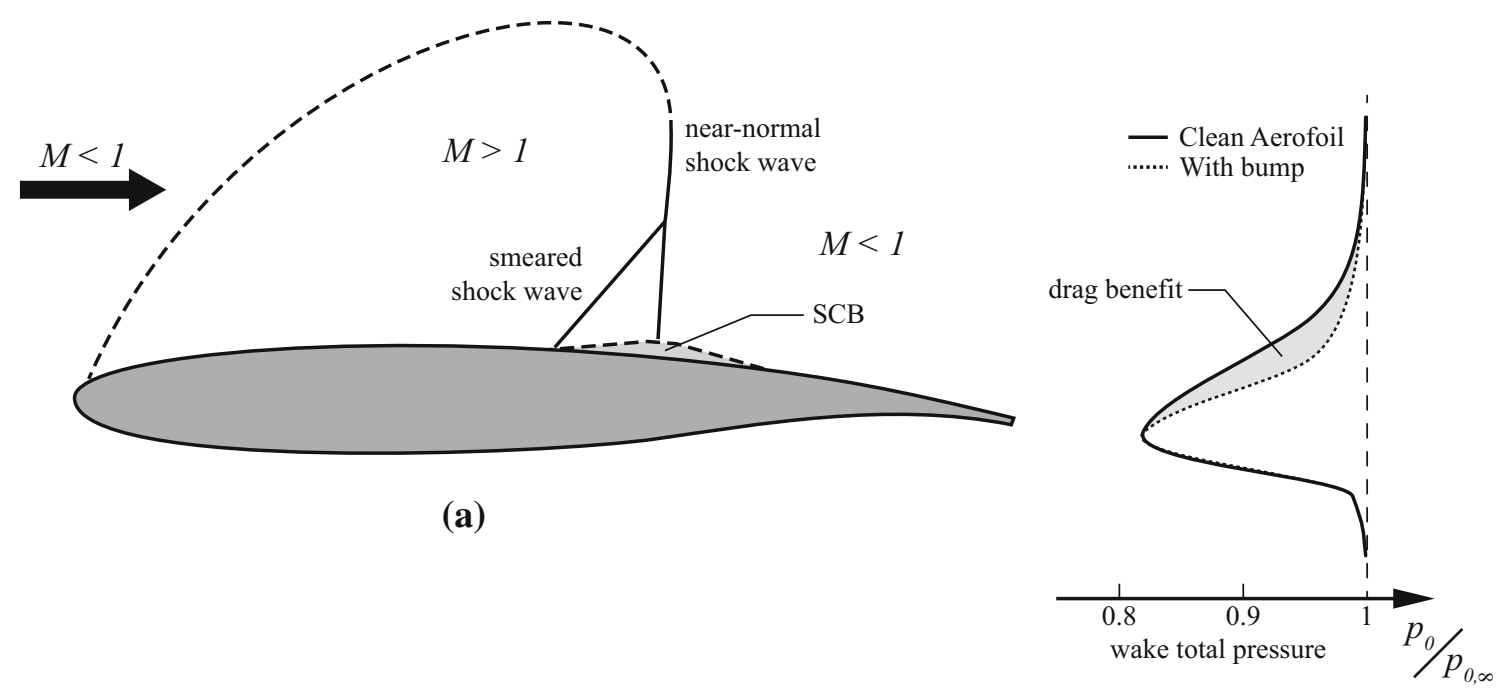

(b)

Fig. 1 Principle of operation of a SCB on a supercritical wing: a transonic flow structure; $\mathbf{b}$ total pressure in the wake

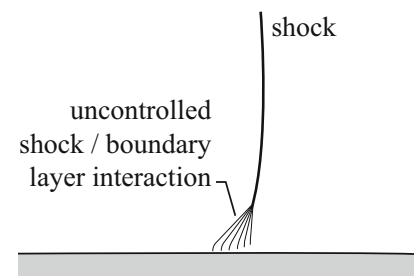

(a)

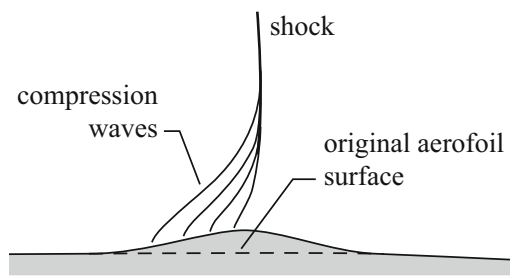

(b)

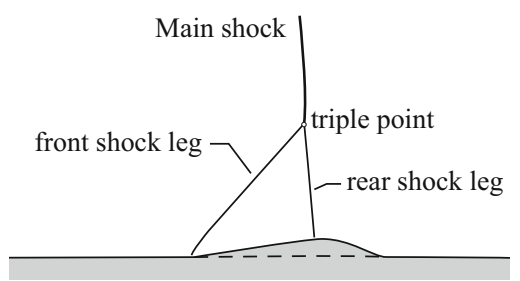

(c)

Fig. 2 Effect of SCBs on shock structure: a no-control case; $\mathbf{b}$ compression waves produced by a smooth contoured bump [4]; $\mathbf{c} \lambda$-shock foot structure produced by a wedge bump (reproduced from [26] with permission of Hideaki Ogawa)

Fig. 3 Illustration of SCB location on aircraft: a 2-D SCB; b array of 3-D SCBs

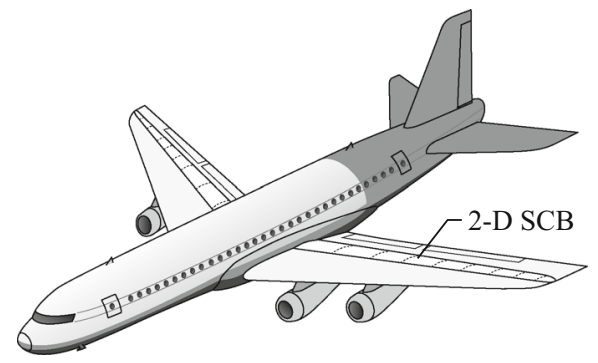

(a)

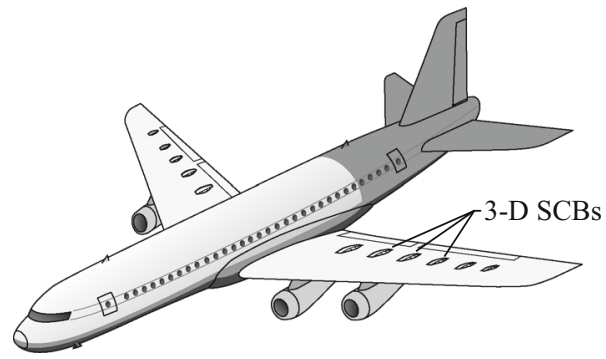

(b)
SCBs can be two-dimensional (2-D), where the bump profile is constant along the wing span or three-dimensional (3D), where an array of finite-width bumps is placed along the wing, as illustrated in Fig. 3. A selection of 3-D geometry SCBs considered by researchers is illustrated in Fig. 4.

\subsection{Basic flow physics}

Range of flow conditions. The flow structures generated by 2-D and 3-D SCBs are highly sensitive to parameters such as (streamwise) shock position and incoming boundary layer state. 3-D SCBs are often considered as an alternative to 2-D devices in response to concerns over the poor performance of 2-D SCBs at so-called 'off-design conditions' (defined in most investigations as being when the shock wave is deemed to be upstream or downstream of its optimal location) $[2,26,29,33]$. This sensitivity necessitates a thorough understanding of the flow characteristics of SCBs over a range of operating conditions, rather than at a single design point, to accurately characterise their practical performance potential. For this reason, data from literature span a large array of test parameters and flow conditions and care is needed to draw together the results and conclusions from complementary studies. Table 1 attempts to summarise the ranges of key 


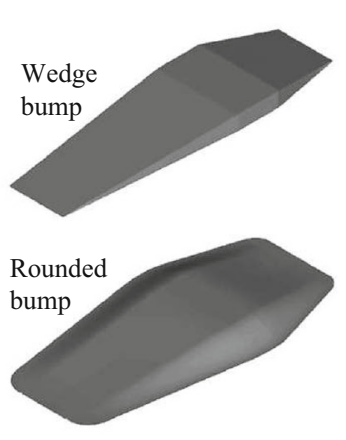

(a)

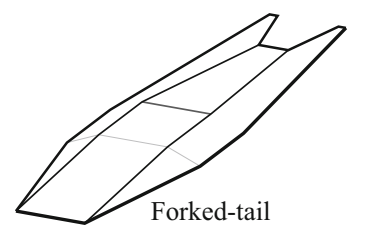

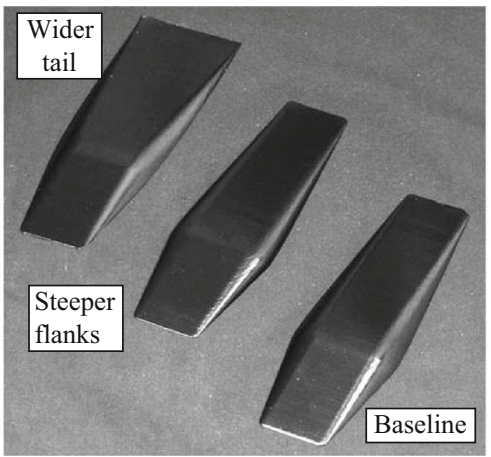

(b)

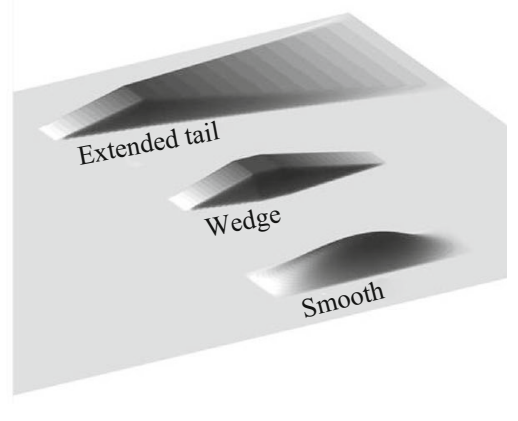

(c)
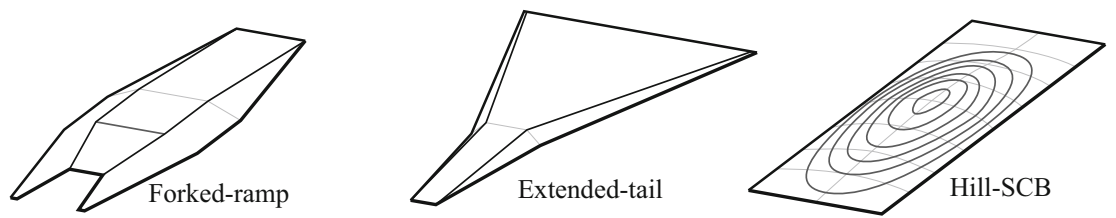

(d)

Fig. 4 Selection of SCB shapes investigated in literature from: a Ogawa et al. [26]; b Bruce and Babinsky [7]; c Colliss et al. [9]; d geometries tested by 'NextWing' programme

Table 1 Range of geometric parameters and flow conditions investigated in SCB literature

\begin{tabular}{ll}
\hline Parameter & Typical values investigated \\
\hline Peak shock strength $(M)$ & $1.3 \pm 0.2$ \\
Ramp angle & $5^{\circ} \pm 2^{\circ}$ \\
SCB height & $\delta_{0} \pm \delta_{0}$ \\
SCB length (length/height) & $15 \pm 5$ \\
SCB aspect ratio (length/width) & $3 \pm 1$ (3D devices only) \\
Shock position & $50 \pm 20 \%$ of SCB chord \\
Test surface & Flat wall, convex wall, \\
& aerofoil surface \\
Incoming boundary layer state & Turbulent \\
\hline
\end{tabular}

geometric parameters and flow conditions investigated by the majority of researchers studying SCBs. Although there are always outliers, it is fair to say that a key message from Table 1 is that most SCB studies have tended to consider bumps with heights of the order of the local boundary layer thickness $\left(\delta_{0}\right)$ and with shock strengths of around Mach 1.3.

2-D SCB: The basic (inviscid) flow structure produced by a 2-D SCB in a transonic flow resembles the illustrations in Fig. 2 (assuming the flow structure is also 2-D). Ashill et al. [2] reported that variations in shock position away from this optimum location gave rise to the appearance of undesirable expansions and secondary shock systems that were detrimental to the performance of 2-D SCBs. Figure 5, adapted from Ogawa et al. [26], illustrates this point, showing the impact of shock position on the flow structure over a 2-D SCB with a simple (straight ramp and straight tail) geometry. This sensitivity of SCB performance to shock position, although not unique to this type of flow control, is a highly prevalent flow characteristic of this type of 2-D SCB and is something that has been confirmed by other researchers $[29,33]$.

The systems of shock waves and expansions illustrated in Fig. 5 impose strong streamwise pressure gradients on the boundary layer that exists on the SCB surface. The behaviour of this boundary layer (i.e. whether it remains healthy, thickens or even separates) is a key factor that determines SCB performance. A significant challenge in SCB design is to achieve beneficial stagnation pressure savings without incurring excessive viscous losses. In general, the flow curvature introduced by a SCB will be detrimental to the health of any incoming boundary layer. Of the three test cases illustrated in Fig. 5, the two 'off-design' test cases (a and c) incur flow reexpansion and secondary shock systems that are detrimental to the boundary layer. In contrast, the optimal shock position (Fig. 5b) yields a 'cleaner' two-step pressure rise which is less detrimental to the boundary layer [26]. Figure 6 presents results from two numerical studies $[10,13]$, both of which illustrate this beneficial impact of a 2-D SCB on the streamwise pressure distribution over the upper surface of a supercritical aerofoil.

Studies have shown that a well-designed 2-D SCB generally has a small detrimental impact on boundary layer health relative to an uncontrolled test case when the shock position is close to optimal. However, non-optimal shock locations yield significantly increased levels of viscous drag and can 


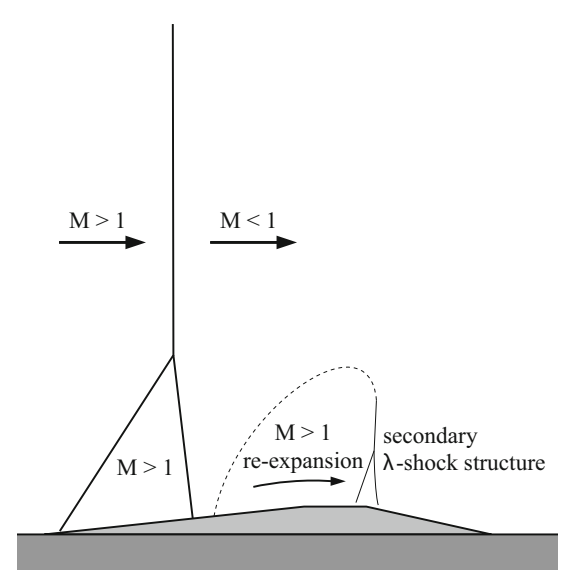

(a)

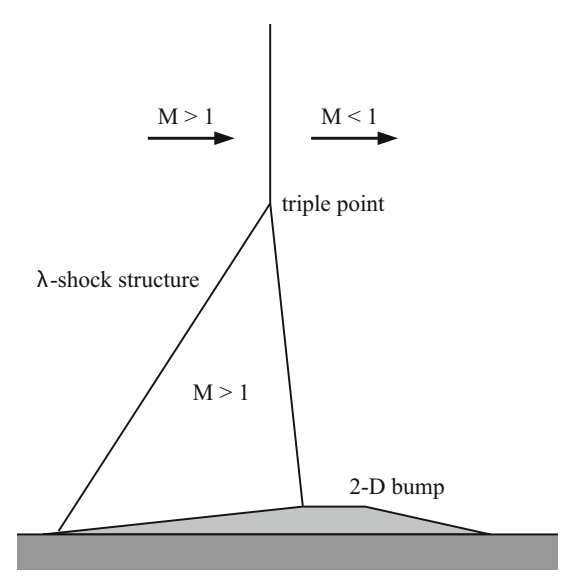

(b)

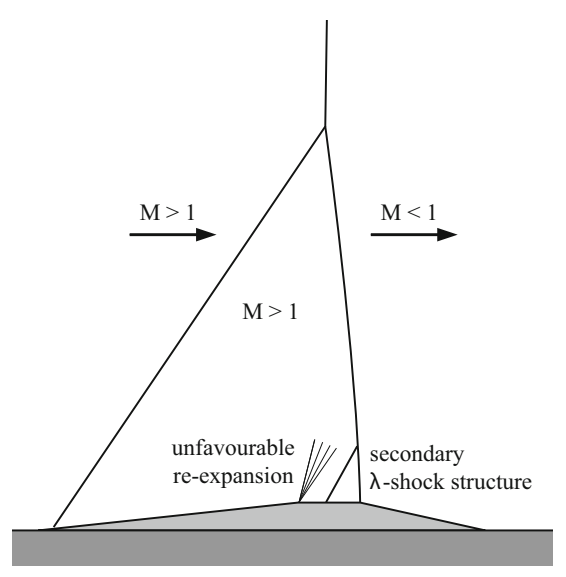

(c)

Fig. 5 Effect of shock position on SCB flow structure, adapted from Ogawa et al. [26] with permission of Hideaki Ogawa: a shock upstream of optimum location; $\mathbf{b}$ at optimum location; $\mathbf{c}$ downstream of optimum location

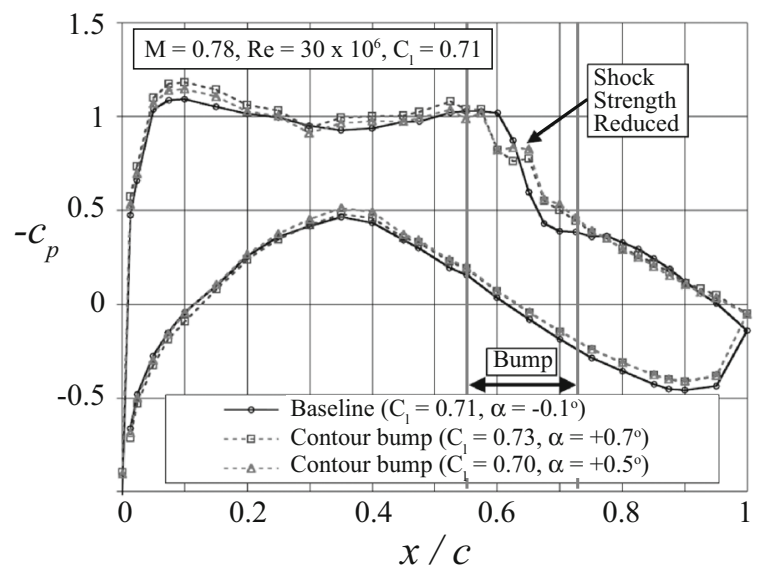

(a)

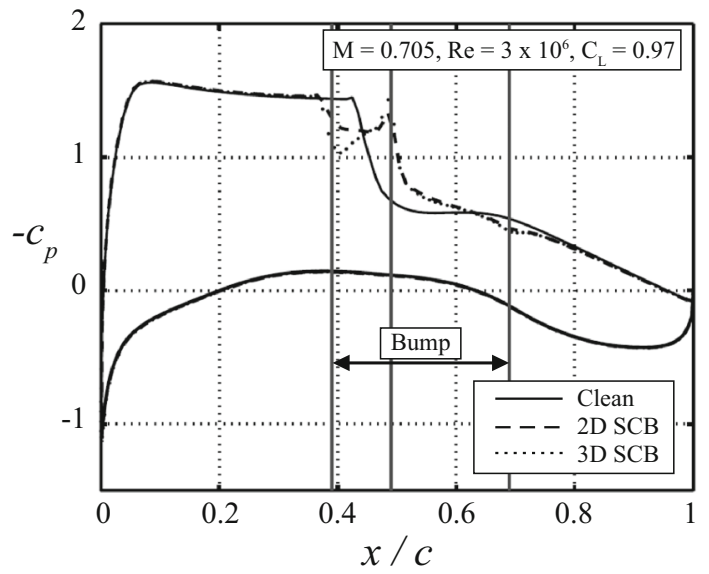

(b)

Fig. 6 Results from: a Milholen and Owens [13]; b Eastwood and Jarrett [10], reproduced with permission from Jeremy Eastwood

also promote boundary layer separation [26]. Despite this, some studies have argued that 2-D SCBs can still have a positive impact on off-design performance in one of the two ways: (1) Delaying the onset of transonic buffet by breaking up large regions of separated flow and inhibiting communication between an oscillating shock and an aerofoil's trailing edge $[2,4,18]$; and, (2) In the case of a straight ramp-type design, improving shock stability by effectively anchoring the front shock leg [17].

3-D SCB: The flow physics of 3-D (finite width) SCBs are different from 2-D devices in a number of ways. In addition to the variables listed in Table 1, the flow physics of 3-D devices are also known to be influenced by SCB width, spacing, taper and flank geometry. 3-D SCBs still generate a (beneficial) $(\lambda)$-shock structure along their central axis but this structure decays in the spanwise direction to produce a curved shock front, as illustrated in Fig. 7a. However, researchers such as
Ogawa et al. [26] have observed that this spanwise decay is quite gradual, such that a spanwise array of carefully spaced discrete 3-D SCBs (spaced so that the shock structures produced by adjacent SCBs overlap) can produce a quasi-2-D beneficial shock structure across the entire span. This effect is illustrated in Fig. 7c.

Figure 7a also highlights the spanwise variation in boundary layer thickness that occurs downstream of a 3-D SCB. In simplistic terms, this effect can be attributed to a 3-D SCB localising its negative impact on the boundary layer to the region directly behind it. However, the real situation is rather more complex, due primarily to the fact that 3-D SCBs have been observed to introduce streamwise vorticity into the flow $[17,26,38]$. The precise mechanism by which this vorticity is generated is still a subject of research, although a recent publication suggesting that the spanwise pressure gradients present on a 3-D SCB are of key importance [9] is perhaps the most convincing to date. 

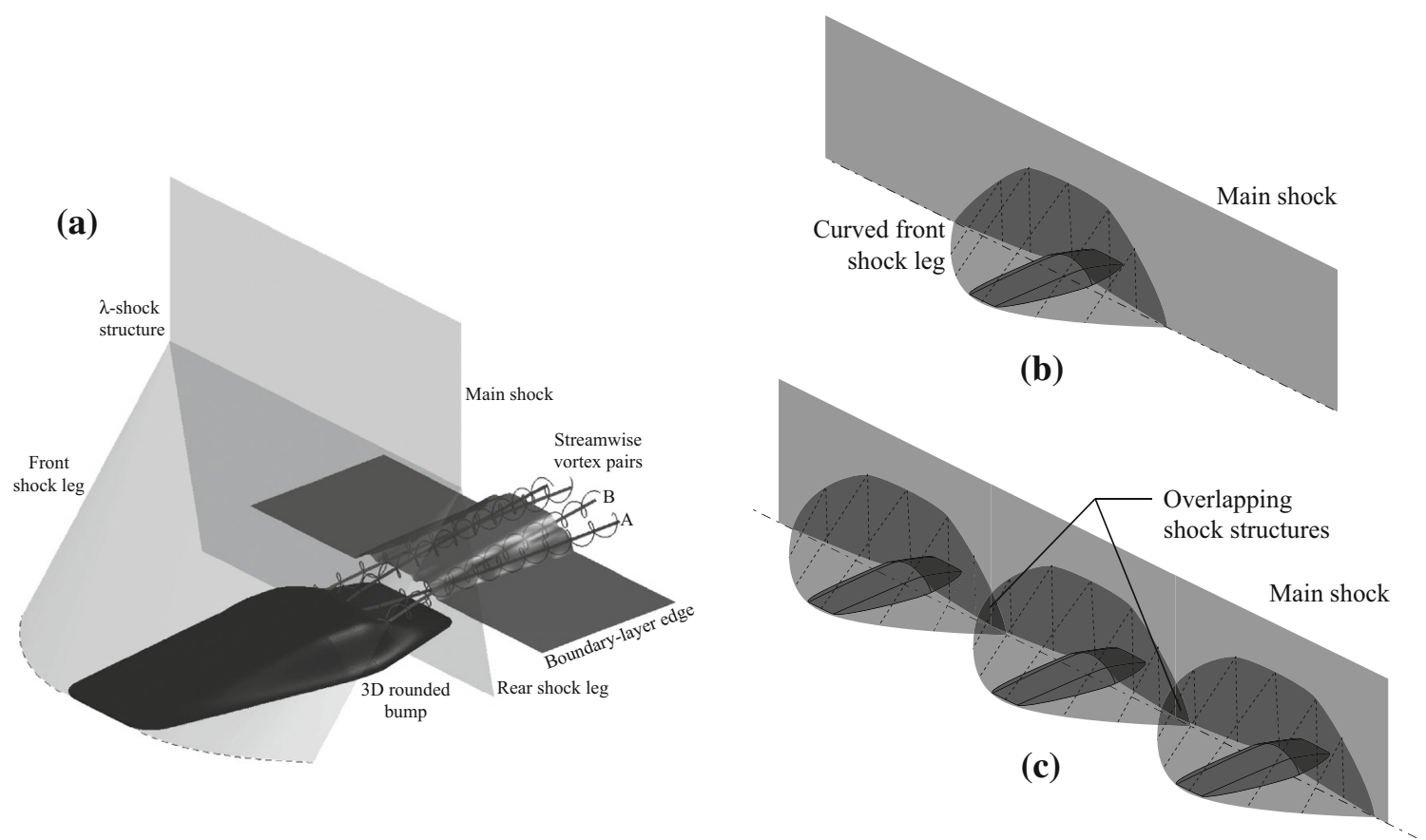

Fig. 7 Spanwise shock structure produced by 3-D SCBs: a shock structure proposed by Ogawa et al. [26]; b spanwise influence of a single 3-D SCB; c 3-D SCB array with overlapping $\lambda$-shock structures. a Reproduced from [26] with permission from Hideaki Ogawa
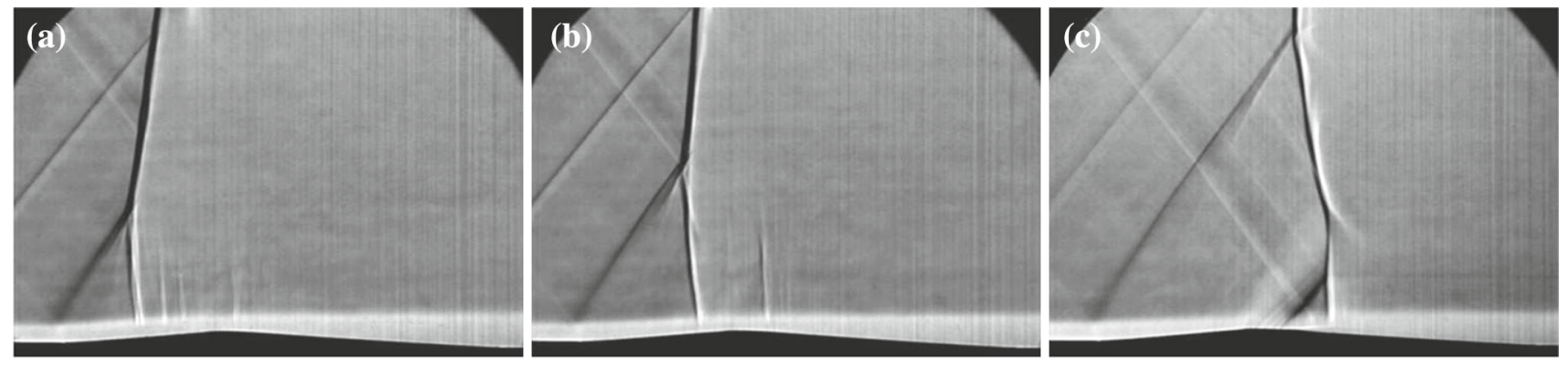

Fig. 8 Schlieren photographs showing impact of shock position on 3-D SCB tested on a flat plate at $M_{\infty}=1.3$ : a shock upstream of SCB crest; b shock close to SCB crest; $\mathbf{c}$ shock downstream of SCB crest. Reproduced from [6]
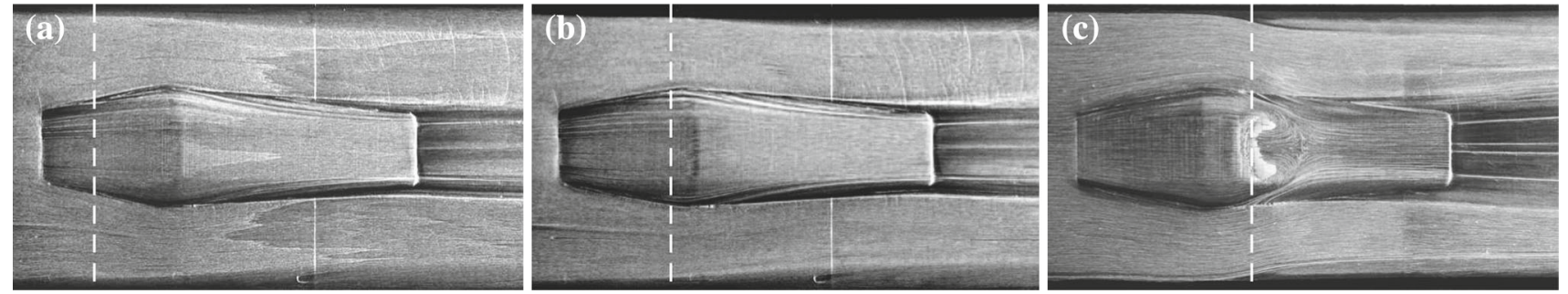

Fig. 9 Surface oil-flow visualisation images showing impact of shock position on 3-D SCB tested on a flat plate at $M_{\infty}=1.3$ : a shock upstream of SCB crest; b shock close to SCB crest; $\mathbf{c}$ shock downstream of SCB crest. Reproduced from [6]

The flow physics of 3-D SCBs can become significantly more complex when the shock moves away from its optimal location. As with 2-D devices, regions of flow re-acceleration and secondary shock structures can appear with a detrimental impact on stagnation pressure recovery (drag) and boundary layer health. Figures 8 and 9 show the impact of shock position on a 3-D SCB tested on a flat plate in a wind tunnel [6]. The surface oil-flow visualisation images in Fig. 9 highlight the complex 3-D flow patterns which include a localised separation bubble near the crest when the shock moves down- 


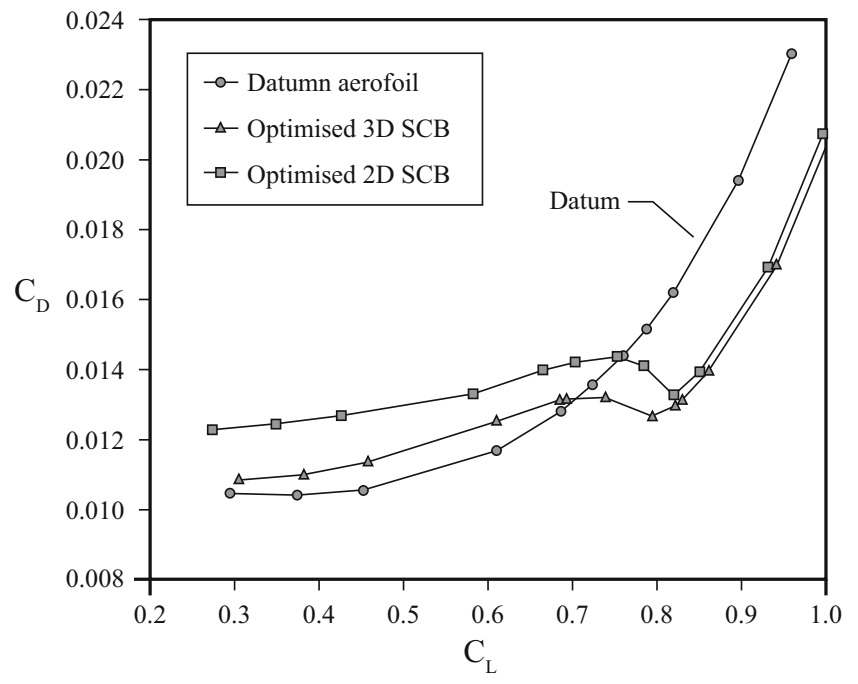

(a)

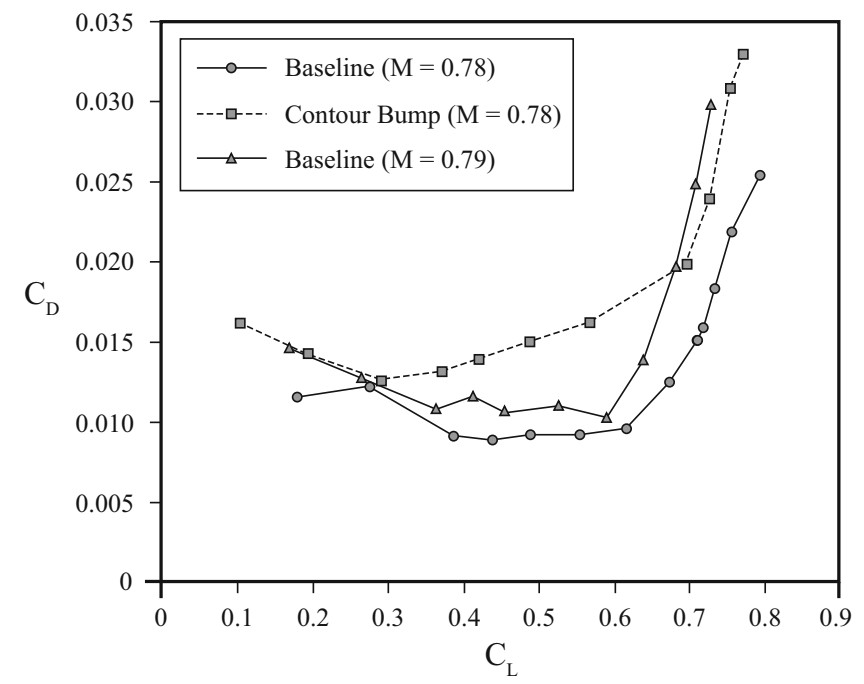

(b)

Fig. 10 Lift-drag polars showing the impact of SCBs on transonic wing performance: a numerical results from Qin et al. [29]; b experimental results from Milholen et al. [13]. a Reproduced from [29] with permission from Ning Qin

stream and dark streaks in the SCB wake indicating the presence of vortical flow structures. The schlieren images in Fig. 8 reveal shock and expansion structures that closely resemble those produced by 2-D SCBs. These apparent similarities are a little misleading as they are due, in part, to the spanwise averaging inherent in the schlieren imaging technique. On the other hand, it does tend to support the observation that 3-D SCBs produce a quasi-2-D shock structure that decays slowly in the spanwise direction.

In comparison with their 2-D counterparts, 3-D SCBs are an especially challenging prospect for CFD.

\subsection{Performance}

In this review, we consider the performance of SCBs in terms of their potential for reducing drag and improving the offdesign performance of transonic wings and aerofoils. In practice, these criteria are most commonly assessed by measuring lift-to-drag ratio $(L / D)$ to assess drag and by studying the boundaries of an aerofoil or wing's performance envelope (i.e. transonic buffet boundary/drag-divergence Mach number) to assess any impact on off-design performance. As a point of clarification, the term 'SCB robustness', commonly used in the literature, is used here to describe the impact of SCBs on aerodynamic efficiency (i.e. $L / D$ ) at non-optimal (off-design) conditions. This is distinct from the ability of a SCB to improve off-design performance as defined in this review; for example, a SCB may have high 'robustness' (i.e. produce high values of $L / D$ at a wide range of conditions) even if it does not yield any benefits in terms of extending a wing's performance envelope (i.e. the buffet boundary and drag divergence Mach number remain unchanged).
Drag. In terms of drag saving potential, SCB performance can be viewed conceptually as a balance between the positive benefits of control (i.e. wave drag reduction) and any negative impacts (e.g. viscous penalty or reduced lift). It is now well-documented that (2-D and 3-D) SCBs can yield a net positive benefit for the efficiency (usually expressed in terms of $L / D)$ of transonic aerofoils and wings $[13,29]$. Two examples illustrating the impact of SCBs on lift-drag polars for different transonic wings are presented in Fig. 10.

Figure 10 highlights a common theme that has emerged from many studies; The performance benefit of SCBs (both 2-D and 3-D) is only realised at high values of $C_{L}$ (and/or $\mathrm{M})$ for conventional (turbulent) supercritical wings or aerofoils. At low values of $C_{L}$, a clean (no SCB) configuration invariably performs better. This trend is consistent with the fact that a SCB can only have a beneficial impact (i.e. reduce wave drag) in cases where a relatively strong shock wave is present. For a modern turbulent supercritical wing, this only occurs at high values of $C_{L}$ and/or Mach number that are in excess of typical cruise values. For this reason, many argue that the true potential of SCBs for drag reduction can only be realised in the long term with an integrated approach where next-generation wings are designed specifically to take advantage of the beneficial control effects offered by SCBs $[13,20,33]$.

Off-design performance. As previously mentioned, the concept of a SCB originated from studies investigating ways to increase the drag-divergence Mach number of transonic aerofoils $[34,35]$. Since then, many studies have considered the 'robustness' of 2-D and 3-D SCBs by testing them at both design conditions as well as a range of off-design conditions 


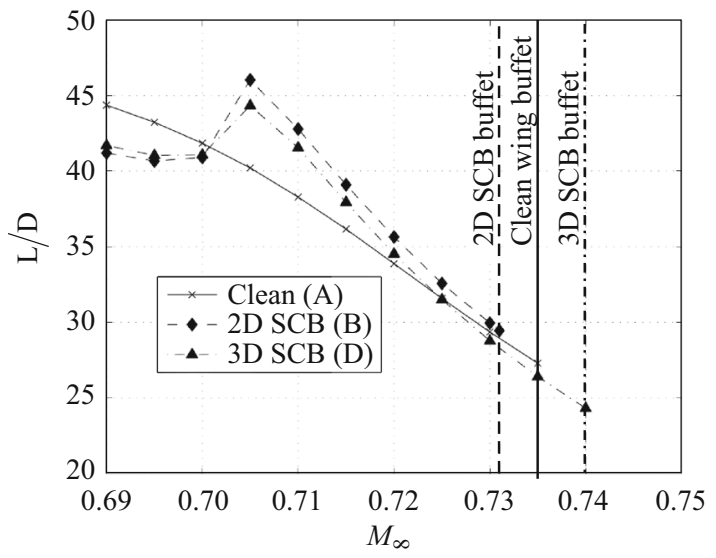

Fig. 11 Buffet boundaries for different SCBs, reproduced from Eastwood and Jarrett [10] with permission from Jeremy Eastwood

representing both near-cruise conditions $[6,26]$ as well as more extreme cases near the buffet boundary [10]. Results from these studies consist of a mixture of qualitative comments and reported measurements (usually $L / D$ ) that quantify the sensitivity of SCB efficiency to flow conditions, such as the results previously shown in Fig. 10.

SCB geometry is known to be an important factor that influences off-design performance: for example, König et al. [17] report that although smoothly contoured SCBs (such as the hill-SCB in Fig. 4) can give optimal drag reduction for the shock at its design point, more angular wedge-shaped devices give improved robustness to variations in shock position with very little loss of efficiency.

Numerous SCB studies have shown that variations in shock position can lead to flow features such as separations and vortical structures (see Fig. 9) which are likely to have an impact on off-design performance of a wing $[6,26]$. Such observations and comments are invariably unquantified; with most studies considering, at best, a small number of 'representative' test cases with different flow conditions. However, one recent study by Eastwood and Jarrett [10] has attempted to address this with a more quantified approach. An element of their study (which is discussed in more detail in Sect. 3 of this review) focused on the prediction of buffet and yielded plots such as Fig. 11, which quantifies the effect of different geometry SCBs on a wing's off-design performance (in this case the position of the buffet boundary). The study confirmed that SCBs have potential to have a beneficial impact in this regard.

2-D versus 3-D. Recently, many studies have focused on comparing the performance of arrays of 3-D SCBs with single 2-D devices [10,17, 26, 27, 29,38]. In general, these studies have shown that, while 2-D SCBs offer the maximum potential benefit in terms of design-point drag reduction for a transonic wing, an array of 3-D SCBs is able to deliver very

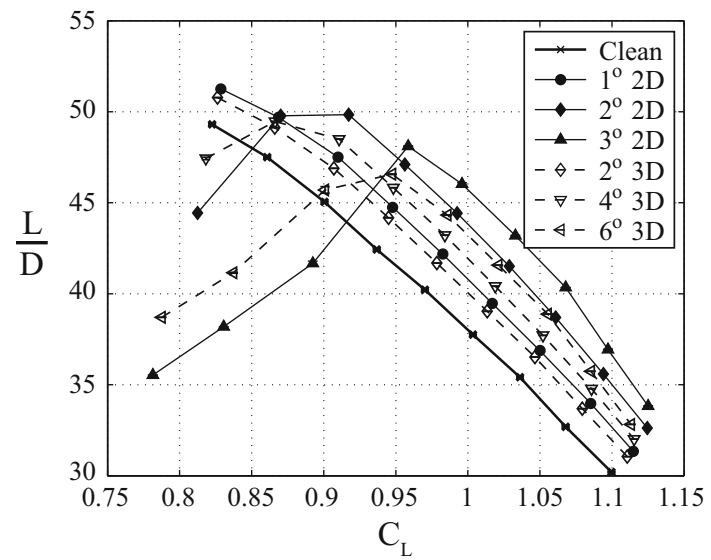

Fig. 12 Comparison of 2-D and 3-D SCB performance on an un-swept transonic wing, reproduced from [10] with permission from Jeremy Eastwood

similar levels of performance benefit (in terms of wave drag saving) with the added advantages of reduced installation cost and complexity and improved robustness to variations in shock position [26,29]. Figure 12 illustrates this trend for a range of different geometry 2-D and 3-D SCBs mounted on an un-swept wing.

An exception to this was the (computational) study by Qin et al. [29], where it was shown that an array of optimised 3-D SCBs actually gave a greater design-point benefit than an optimised 2-D SCB for an un-swept natural laminar flow wing (results shown previously in Fig. 10a). This result brings into question whether the supposed '2-D limit' on designpoint performance for 3-D SCB arrays may be misleading and overly simplistic. Even if this single exception to the rule is disregarded, it is still significant that 3-D SCB arrays can get very close to the performance of 2-D devices, while producing distinctly different flow physics. In this context, it is reasonable to conclude that understanding the flow features unique to 3-D SCBs (including vortical flow structures and spanwise variations in boundary layer properties) will play a key role in unlocking their full potential.

\section{Detailed flow physics: recent advances in understanding}

In the last decade, there has been a resurgence of interest in SCBs and significant progress in our understanding (especially of 3-D devices). In this section, we focus on progress in four areas: (1) The design of experiments for studying SCBs; (2) The effects of flow conditions on SCB performance (including shock position, shock strength, boundary layer state and post-shock pressure gradient); (3) The impact of 3-D SCB geometry on performance; and (4) The generation of vorticity by $3-\mathrm{D}$ SCBs. 
Fig. 13 Typical pressure distributions through transonic SBLI for wind tunnels and aerofoils, adapted from [8]

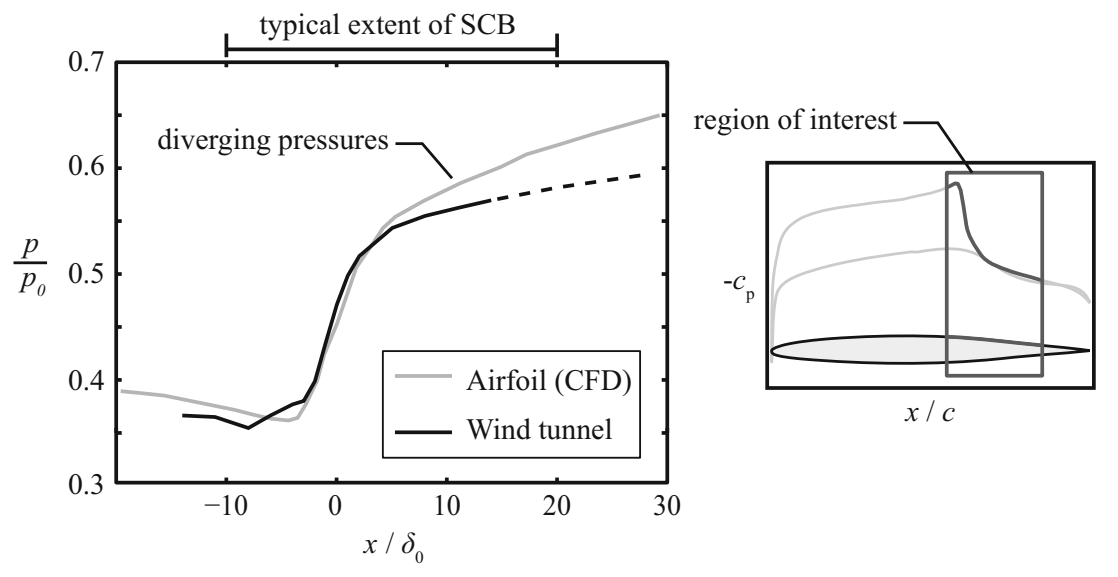

\subsection{Design of experiments for SCB studies}

In designing experiments to study the details of SCB flow physics, the most basic requirements are that

(i) experiments are repeatable and relatively inexpensive,

(ii) the bumps are sufficiently large to enable their flow structures to be resolved by the experimental techniques,

(iii) the flow to which the bumps are subjected is relevant to an aerofoil or wing.

The first of these is necessary to ensure that the flow can be examined using a variety of techniques with confidence that the flow features observed are the same each time. If a test is expensive to perform, then this will severely limit the number of times it can be run and, as a consequence, the amount of data that can realistically be gathered. The second requirement is of obvious importance given the aim of the experiments; whilst the third is critical to ensure that the flow physics observed can be expected to occur if the bump was placed on a wing. Unfortunately, these three conditions typically conflict with one another.

A number of experiments have been performed in the past where a SCB was mounted on a wing model in the wind tunnel-for example, König et al. [17], as well as a number of unpublished tests. This clearly deals with the issue of representativeness of the baseline flow, and offers other advantages such as the ability to directly measure the effect on lift and drag, as well as having a shock strength which varies with operating condition, which is more realistic of wings. However, these tests have typically been found to be difficult to set-up as well as expensive. In addition, the size of the wind tunnel dictates the size of the wing that can be tested, and results in small SCBs. This prevents the resulting data resolving the smallest scale details of the flow. In addition, some authors have reported that the results obtained in such environments are not entirely convincing anyway; König et al.'s study found that there were significant wall interference effects that limited their validation to computations modelling the confined channel-this was therefore not fully representative of an aircraft in free flight.

It is therefore unsurprising that much of the experimental work that has been performed on SCBs has been done using bumps mounted on the floor of small-scale supersonic wind tunnels-notably $[6,8,9,12,26,38]$. The advantage of this is that the tests are inexpensive, repeatable and concentrate solely on the flow local to the bump, thus enabling a detailed examination. The principal disadvantage is that the baseline flow does not look much like that on an aerofoil: Fig. 13 demonstrates this problem with respect to the surface pressure distribution through the shock/boundary layer interaction, which typically has a significantly higher post-shock adverse pressure on an aerofoil than in the wind tunnel experiments. A second area in which the small-scale wind tunnel is likely to be unrepresentative is the state of the boundary layer ahead of the bump, since they are grown in significantly different environments on the wing and in the tunnel, [8]. Finally, there is likely to be significant impact of the wind tunnel aspect ratio, which at best limits the effective bump spacing and at worst introduces unwanted three-dimensionality into the flow.

A further problem with such wind tunnel experiments is that they cannot give any indication of how well the bump performs in terms of drag reduction, as well as failing to provide relevant validation data for computational studies. Earlier combined studies, for example, that of Wong et al. [38], addressed the latter using CFD to model the wind tunnel flow directly. Whilst this enabled the CFD to be appropriately validated, it did not satisfy the need for performance indicators.

Recent work by Colliss et al. [8] has defined a joint experimental and computational approach to SCB investigations. By modifying the working section of a blow-down supersonic wind tunnel to increase the post-shock adverse pressure gradient and enable manipulation of the incoming bound- 
Fig. 14 Comparison of experimental surface oil flow and numerical surface streamlines, adapted from [8]: $\mathbf{a}$ hill SCB; $\mathbf{b}$ extended tail SCB

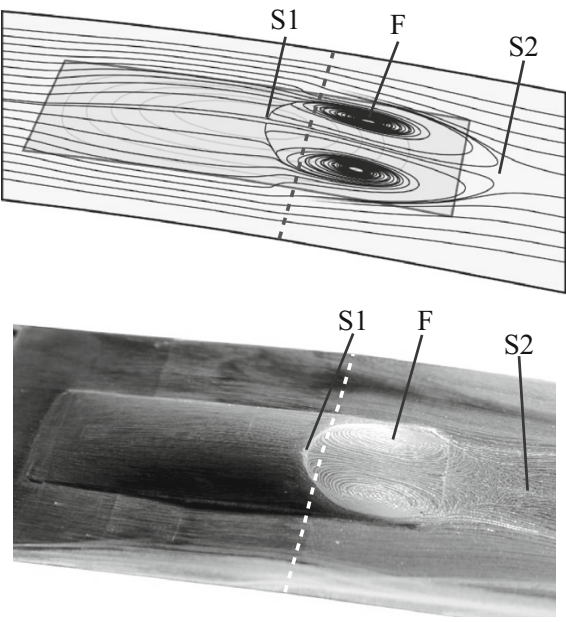

(a)
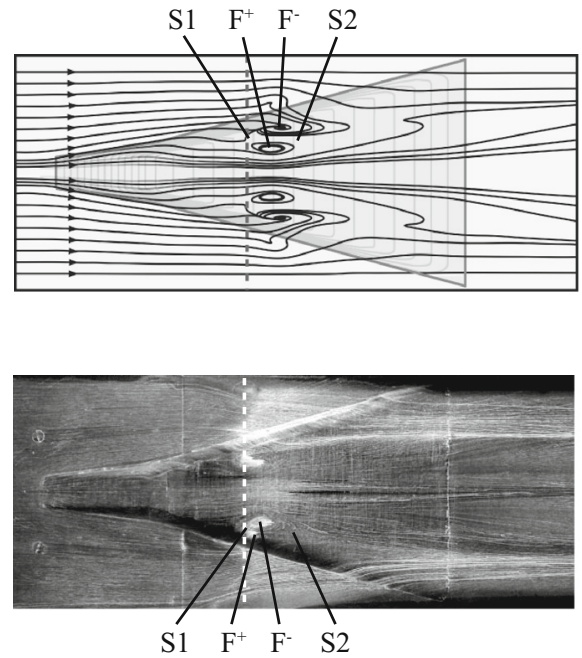

(b) ary layer, they demonstrated that the wind tunnel can produce conditions which are representative of the flow on an unswept wing. Furthermore, in spite of the small aspect ratio of the wind tunnel, provided the bump array spacing in the CFD is chosen accordingly, the experiments and computations still produce very comparable results—even for bumps which span a significant proportion of the tunnel widthFig. 14. A key advantage of this joint approach is that it also allows the performance of a bump and the flow around it to be correlated - additional (as yet unpublished) work from the same investigation is aiming to examine the aspects of the flow which indicate the bump's potential for $L / D$ improvement.

\subsection{Effect of flow conditions on SCB performance}

Often the flow conditions which affect SCB performance are not independent. This is especially true in real aerofoil geometries where, for example, shock position, shock strength and post-shock pressure gradient are intrinsically linked. In this section, we attempt to give some impression of the comparative importance of a number of different flow condition variables. To achieve this, we focus primarily on studies in carefully controlled environments and attempt to identify consistent trends between such tests.

Boundary layer: Properties of the incoming boundary layer such as its fullness (shape factor, $H_{i}$ ) and thickness are known to have an impact on the flow over a SCB. The consensus from literature is that SCBs with heights of the same order of magnitude as the incoming boundary layer thickness perform strongly. If the boundary layer is much thicker than the SCB, the beneficial shock-smearing effect of the control device is significantly weakened. On the other hand, tests with bumps that are significantly taller than the incoming boundary layer thickness can produce promising results [26], although there are other disadvantages with such designs, especially in offdesign conditions. In practice, this rule-of-thumb for bump height approximately equal to boundary layer thickness has proven to be well matched to the practical constraints of mounting a SCB on a real wing (where SCB height is indirectly limited by the need to bring the surface of the SCB back down to the aerofoil without unacceptably high flow turning angles).

Although SCBs have long been considered as a candidate for use on natural laminar flow wings (where shock strengths are necessarily higher), the performance of a SCB in laminar flow has not been investigated. This is because laminar boundary layers invariably separate in the presence of even weak shock waves, which would yield poor performance. For this reason, it is necessary to force transition some distance ahead of the SCB on laminar flow wings. McIntosh and Qin [21] performed simulations where they artificially fixed the transition location at different (chord-wise) positions in the range $0-45 \%$ chord. Their results showed that SCB performance was largely insensitive to transition location, although variations in boundary layer displacement thickness did affect the position of the rear $\lambda$-shock leg slightly.

Shock position: Figures 8 and 9 highlight the strong sensitivity of SCB performance to shock position. The wind tunnel tests that produced these images (single 3-D SCB mounted on a flat plate at $M=1.3$ ) showed that variations in shock position around the SCB crest of as little as $10-15 \%$ of SCB length caused abrupt changes in the nature of the flow, including the appearance of a significant separated region (evident in Fig. 9c). If such a SCB was installed on a real wing with length of the order of $20-30 \%$ wing chord - a range commonly suggested in literature $[10,13]$ — such variations in 

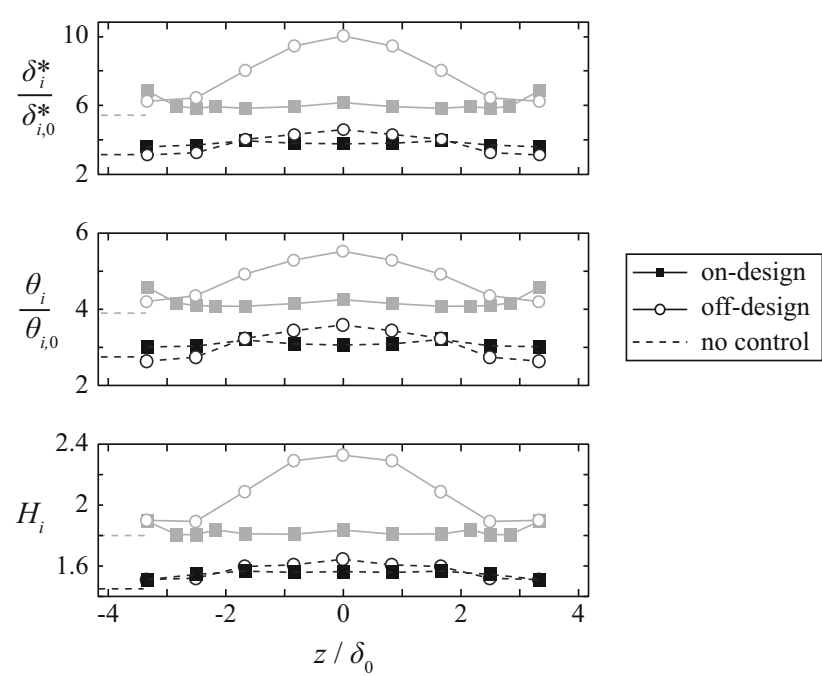

Fig. 15 Effect of streamwise pressure gradient on boundary layer downstream of a wedge SCB: dashed dark grey lines, [7]; solid light grey lines, SCB using set-up of [8]

shock position would correspond to excursions of just 2$4 \%$ of overall wing chord. These numbers suggest that the impact of shock position on SCB performance is a primary concern.

Shock strength: Relatively few studies have explored the effect of varying shock strength in isolation, in part because performing such studies (especially experimental ones) is very challenging. Such studies are only possible in parallel walled ducts where the incoming (supersonic) Mach number is uniform and set by an upstream throat and the shock position is fixed artificially. Babinsky and co-workers at Cambridge University spent considerable effort developing such a set-up [25] that allowed them to perform studies with SCBs at two discrete Mach numbers: 1.3 and 1.5 [3, 6, 12,24,26,38] and also a small number of (currently unpublished) tests at Mach 1.4. A common conclusion from these studies was that higher Mach numbers caused greater levels of separation that promoted stronger vortical flow structures in the wakes of 3-D SCBs. Eastwood and Jarrett [10] investigated the impact of shock strength on the performance of 2-D and 3-D SCBs on a real wing geometry by performing computations at a range of conditions (variable $M_{\infty}$ and $\alpha$ ) that maintained the same shock position with different shock strengths. They showed that variations in shock strength in the range $M=1.18-1.38$ had a significant impact on the performance (in terms of $L / D$ ) of the wing with or without SCBs. This is consistent with a general breakdown of the flow in the presence of strong shock waves when significant shockinduced separation occurs. Their results suggested that all SCBs tested gave a benefit when the shock strength exceeded approximately $\mathrm{M}=1.27$. For shock strengths above this value, 2-D and 3-D SCBs with steeper ramps (and hence larger overall heights) performed marginally better, within the range of geometries tested. For shock strengths below 1.27 , the opposite trend was true, with less high SCBs giving better performance.

Pressure gradient: Figure 15 shows the spanwise variation of the kinematic integral boundary layer parameters downstream of a wedge bump. The data shown are adapted from the study of Bruce et al. [7] for a flat plate wind tunnel, and data taken for the same SCB geometry using the wind tunnel set-up of Colliss et al. [8]. Whilst the general behaviour of the bump under design conditions is very similar-with the larger values of $\delta_{i}^{*}, \theta_{i}$ and $H_{i}$ being observed also in the uncontrolled flow - the off-design behaviour is significantly worse in the presence of a streamwise pressure gradient. This is due to the separation extent being larger in the presence of a higher post-shock adverse pressure gradient, as shown in Fig. 16.
Fig. 16 Effect of streamwise pressure gradient on shock-induced separation topology: a flat plate, from [7]; b data from the set-up of [8]

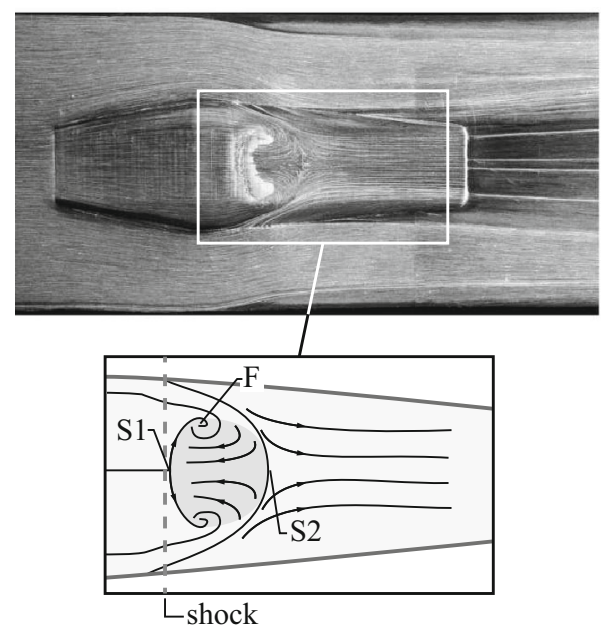

(a)

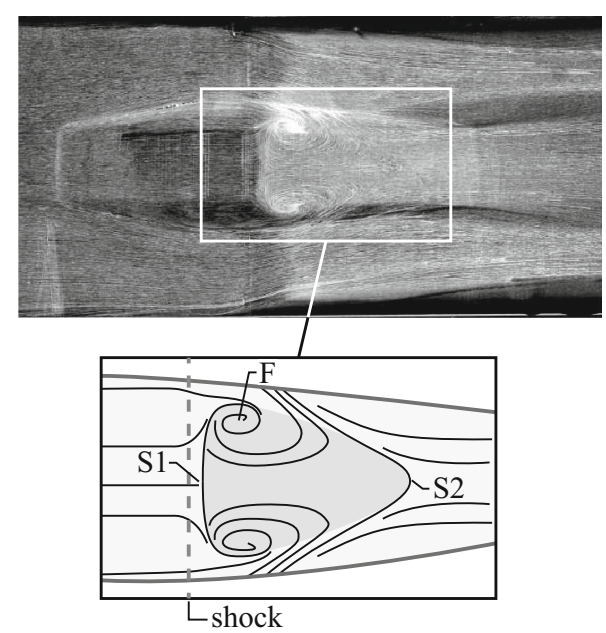

(b) 
Fig. 17 Wake structure visualised by contours of wall-normal velocity $20 \mathrm{~mm}$ downstream of a wedge SCB: a flat plate data from [7]; $\mathbf{b}$ data from the set-up of [8]

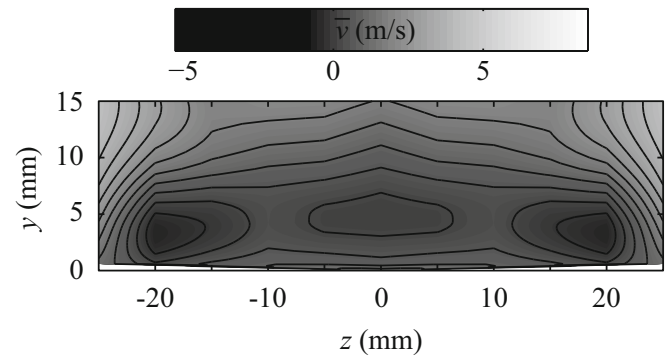

(a)

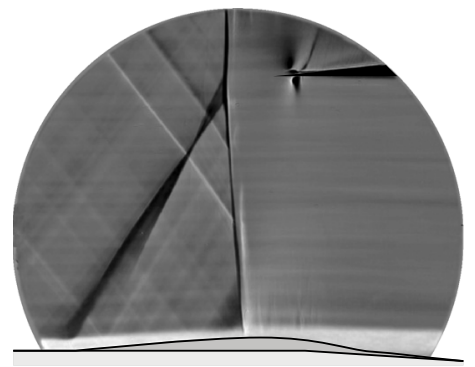

(a)

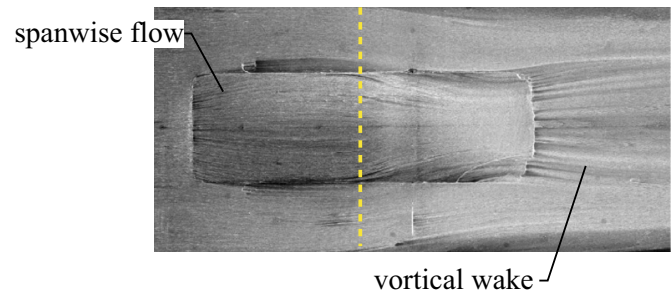

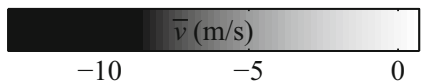

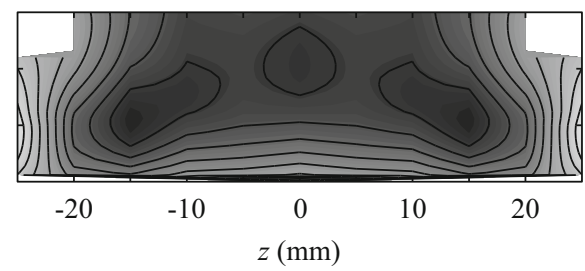

(b)

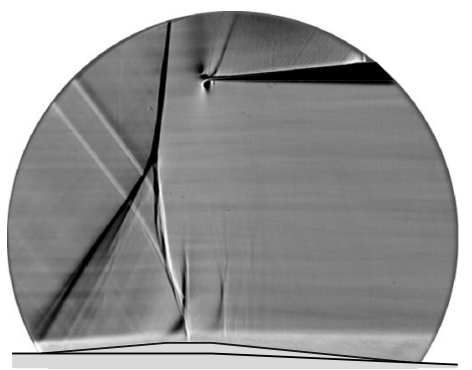

(b)

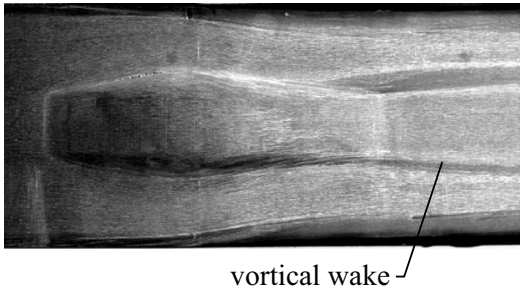

Another effect of the streamwise pressure gradient can be seen by considering the wake structure. Figure 17 shows the wall-normal velocities for wedge bumps in the set-ups of $[7,8]$. The vortices in both (visible from the down-wash patches in both) are seen to be similar strength relative to the background flows; however, the data from [8] have the vortices further from the wall and slightly closer to the bump centreline than that from [7]. Whilst this maybe due to sidewall effects being larger in the case with additional pressure gradient, the measured vortex position agrees well with computations on an aerofoil, suggesting that perhaps this is genuine. Additionally, there is a stronger down-wash in the centre, which extends higher up from the floor in Fig. 17b, suggesting a more significant flow distortion due to the bump.

\subsection{Effect of geometry for 3-D SCBs}

The performance of different geometry SCBs, including those in Fig. 4, has been studied in tests on empirically derived shapes in controlled environments $[7,8,26]$ and also more systematic optimisation-style studies involving tests on parametric (scaled) versions of baseline geometry SCBs, for example $[10,27,29]$. In this review, we do not attempt to assess every shape ever tested but instead aim to summarise common points that are applicable to SCBs in general. We limit ourselves to considering 3-D devices, which we divide into two main categories: (1) Wedge-like geometries, which have a distinct ramp-crest-tail design, like the majority of shapes in Fig. 4; and (2) Smooth contoured designs, such as the smooth bump and hill-SCB in Fig. 4. Figure 18 shows results from wind tunnel tests comparing the principle flow features over these two classes of SCB.

\subsubsection{Wedge-shaped SCBs}

Bruce and Babinsky [6] described the flow over wedgeshaped SCBs by considering separately the flow over the ramp, crest and tail. We adopt the same approach here and in addition we consider the impact of SCB flank (side) geometry.

SCB ramp: Ramp geometry defines the beneficial smeared shock structure produced by a SCB but does not have a significant impact on boundary layer health $\left(H_{i}\right)$ downstream of a bump. Unsurprisingly, 3-D SCBs with wide ramps produce quasi-2D $\lambda$-shock structures. Highly three-dimensional 

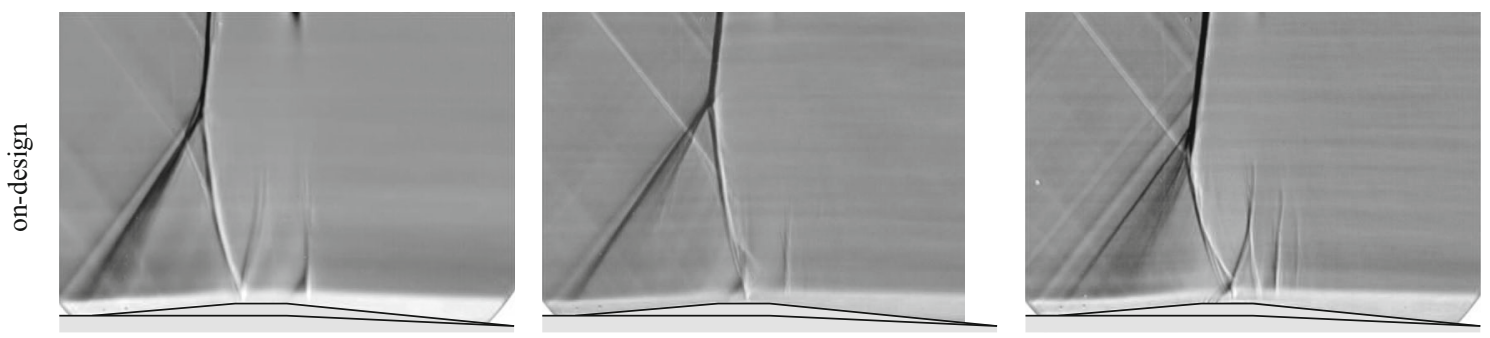

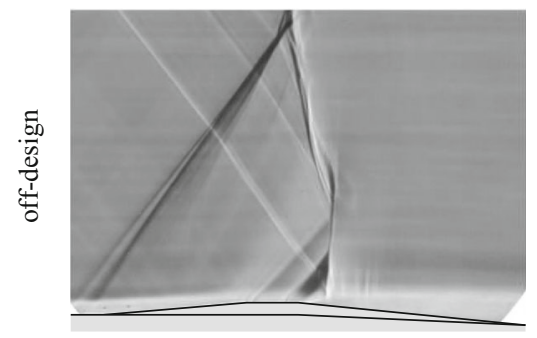

(a)

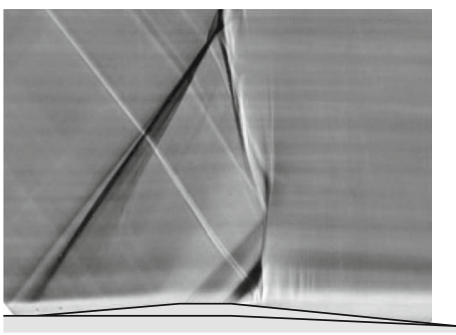

(b)

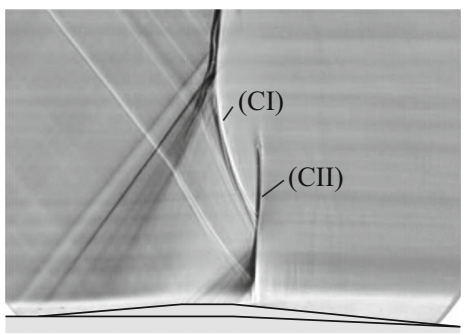

(c)

Fig. 19 Schlieren images of shock structure for forked SCBs: a wedge bump, following [7]; b forked-tail SCB; c forked-ramp SCB

ramps (such as the forked-ramp in Fig. 4d) generate a complex non-uniform shock structure (see Fig. 19c) which is detrimental to total pressure recovery and promotes high levels of distortion in the bump wake. Ramp geometry can impact local separation topology, especially at off-design conditions, by changing the local (streamwise) pressure gradients. The sharp leading edge of a wedge-shaped SCB is known to improve shock stability (relative to a smoothly contoured SCB) by anchoring the front leg of the $\lambda$-shock system. Spanwise pressure gradients across the ramp are thought to play an important role in the generation of vorticity by a SCB. This is discussed further in Sect. 3.4 of this review.

$S C B$ crest: The convex curvature associated with the crest on a wedge-shaped SCB is often greater than that on a comparable smooth-contoured design, due to the more abrupt transition from ramp to tail. This high level of curvature can cause significant local wall-normal pressure gradients and re-acceleration of the flow. For on-design test cases (when the rear leg of the $\lambda$-shock sits close the SCB crest), the flow onto the SCB crest (leaving the ramp) is invariably supersonic. Although the rear leg of the $\lambda$-shock decelerates this flow to a nominally subsonic velocity (very close to Mach 1), high levels of crest curvature can quickly re-accelerate this post-shock flow to produce secondary supersonic regions on the SCB tail with additional associated shock waves.

This concentrated region of high curvature makes wedgeshaped SCBs especially sensitive to small variations in shock position about the crest. Even very small downstream movement of the shock wave past the crest can lead to very strong re-acceleration of the flow to high local Mach numbers. This can cause the rear leg of the main $\lambda$-shock structure to become very strong and provoke boundary layer separation. Gentle rounding of the crest can reduce this undesirable behaviour, as adding a flattened section downstream of the nominal rear$\lambda$-shock leg position so as to give the post-shock flow some distance to recover and decelerate further before it is required to turn back towards the surface.

SCB tail: The tail of a wedge-shaped SCB has a significant effect on boundary layer health and relatively little impact on the shock-structure and associated total pressure recovery in the bump wake. In particular, SCB tail length has been shown to be a critical parameter: SCBs with longer tails (such as the extended tail SCB in Fig. 4) produce healthier boundary layers than equivalent SCBs with shorter tails (as can be seen in the plots of shape factor $H_{i}$ in Fig. 20). The extended tail SCB also gives a performance benefit at off-design conditions, with separation delayed until much larger downstream shock displacements.

Recent (currently unpublished) simulations by Qin and co-workers at Sheffield University suggest that tail width can also be important, and that a flared tail with a flare angle of around $30^{\circ}$ gives a significant reduction of viscous drag at on-design conditions. There is also evidence [7] that a wider tail can be beneficial at off-design conditions by reducing reacceleration over the crest to obtain a reduction in the extent of local separation around the SCB crest region. The forkedtail design in Fig. 4d was observed to give some localised benefit to the boundary layer downstream of the forks but did not change the separation topology (off-design). Vortical structures on bumps with modified tails were similar, suggesting that tail geometry might not be as influential in this respect as was once thought [6]. 

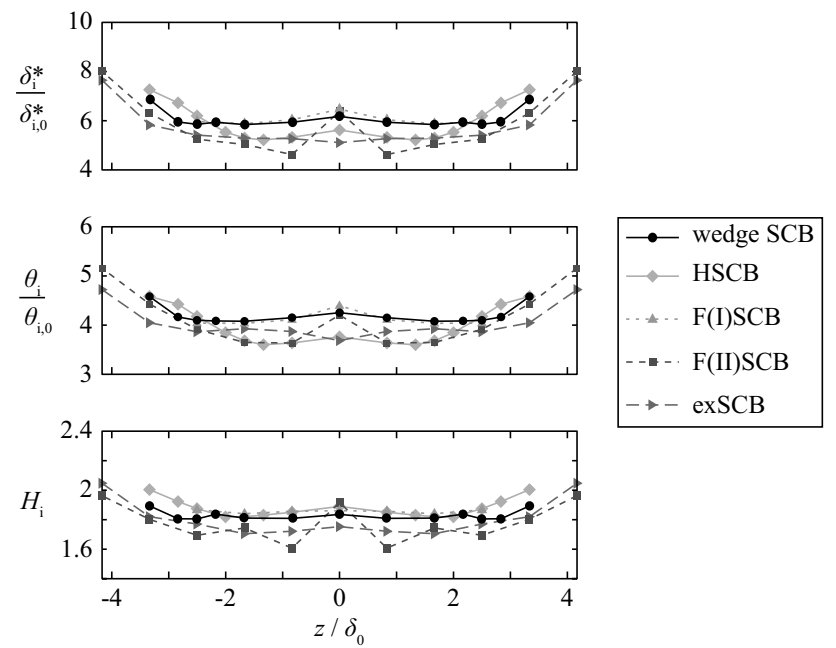

Fig. 20 Kinematic integral boundary layer parameters downstream of SCBs tested in NextWing program, measured across span at $x / \delta_{0}=$ 28.33. $\delta_{i}^{*}$ and $\theta_{i}$ are plotted normalised by the values of these parameters in the undisturbed incoming boundary layer just upstream of the device $\left(\delta_{i, 0}^{*}\right.$ and $\theta_{i, 0}$ respectively)

SCB flanks: Early investigations with 3-D SCBs considered coffin-type geometries with sharp vertical sides [12]. Ogawa et al. [26] showed that the use of rounded flanks improved total pressure recovery and speculated that such designs would perform better in skewed flow. Tests on SCBs with different flank angles (as depicted in Fig. 23) suggest that flank angle on the SCB ramp can impact performance while flank angle on the SCB tail has little effect [7]. It is speculated that spanwise turning of the flow (as the SCB gets wider along the length of its ramp) is increased as the ramp sides are made steeper, giving a $\lambda$-shock structure that propagates over a greater spanwise extent and is essentially more 2-D.

\subsubsection{Contour bump}

König et al. [17] claim that smoothly contoured SCBs, such as the hill-SCB, can give marginally better on-design drag reduction performance than wedge geometries but are less robust off-design. This poor robustness stems from the extensive region of high convex curvature that starts some distance upstream of the crest and leads to strong re-acceleration of the flow and strengthening of the rear shock leg for any rearward shock positions. Extensive re-acceleration of the flow over a smooth contour bump can be seen in Fig. 18, where it can also be observed that such a SCB gives a considerably taller $\lambda$-shock structure than an equivalent wedge bump with the same planform dimensions. Thus, performance of smoothly contoured SCBs can be viewed as a trade-off between an increased area of beneficial shock smearing (which stems from the concave upstream part of the bump) and undesir- able strengthening of the rear shock leg (from re-acceleration of the flow over the extensive convex part of the bump). Figure 20 shows that the hill-SCB is marginally more detrimental to boundary layer health than an equivalent wedge-shaped device.

\subsection{Generation of vorticity}

A prominent flow feature associated with 3-D SCBs is the vortical wake structure. Whilst the most striking vortex formation is caused by flow separation when the SCB is operating under off-design conditions, the bumps also produce vortices when operating under design conditions which are not only observable, but also produce measurable effects on the downstream flow $[6,8,9,24,29,38]$. In its guise as a drag reduction device, it can be argued that the presence of streamwise vortices is not welcome, since it will add a (small) element of drag. However, in reality the situation is more complex because the nature of the vortex pair could actually exert its own boundary layer control. Indeed, the role of SCBs as a novel class of 'smart' vortex generator is beginning to be explored $[9,10]$.

The generation mechanism for off-design conditions is clear from surface oil flow visualisations, with the separation topology including pairs of spiral nodes (or foci), from which a vortex will emanate. Examples of these are shown in Fig. 14, which are typical of SCBs at $M \sim 1.3$ throughout the literature, and produce a pair of common-flow-up counter rotating vortices. However, this vortex pair tends to decay relatively rapidly downstream, and a stronger common-flow-down vortex pair is seen to emerge (Fig. 21a) which causes measurable improvements in the boundary layer relative to the no-control case in spite of the flow separation (Fig. 21b). Some further research from a collaboration between Cambridge University and IAG at Stuttgart, which is currently unpublished, has suggested that the extent to which the common-flow-down vortex pair is able to dominate the wake is a function of the bump spacing, with narrow spacings resulting in a stronger centreline downwash.

The mechanism of the on-design generation of vorticity is more elusive, with a number of alternative suggestions throughout the literature: Wong et al. [38] suggested that separation along the side flanks of the bump leads to a vorticity sheet which rolls up into the streamwise vortex observed. However, their bump had sharp edges down both sides, which would make such a flow structure more viable than on the smoother bump shapes of other investigations. Bruce and Babinsky [6], suggest that the vortex production on a wedge bump occurs at the bump crest under the influence of the local spanwise pressure gradients. Eastwood and Jarrett [10], propose a mechanism of competing spanwise pressure gradients causing a shear layer to wrap up into a vortex pair towards the end of the bump tail. Measurements of Colliss et al. [8], show 
Fig. 21 Wake characteristics of a separated SCB flow, adapted from [9]: a streamwise vorticity structures; $\mathbf{b}$ effect on total pressure in a spanwise plane $30 \delta_{0}$ downstream of the bump trailing edge. Total pressure contours were obtained using an array of Pitot probes

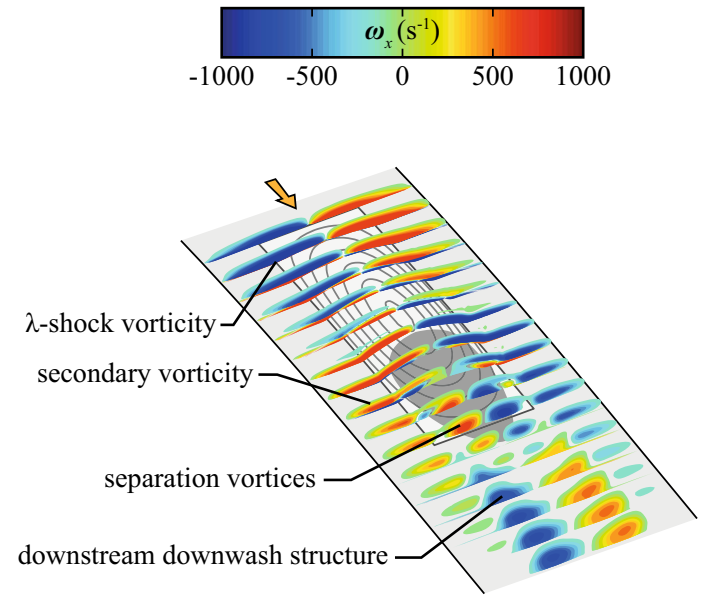

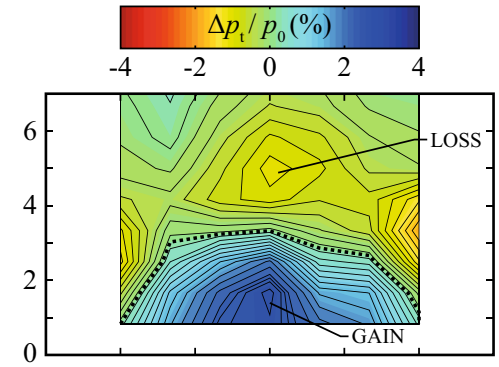

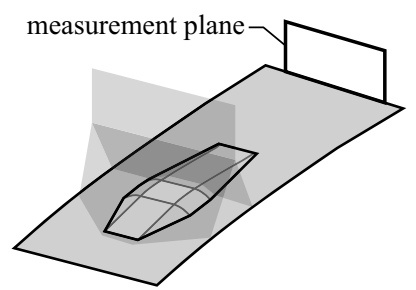

(a)

(b)

the vortical structure developing near the centreline of a contour bump and increasing in magnitude towards the tail before decaying in the wake. Further work by the same authors [9], has identified a number of vortical structures produced by SCBs which appear to be true of any bump geometry:

(i) ( $\lambda$ )-shock vorticity, caused by shear flows over the bump ramp; no vortices are detected in this region,

(ii) secondary tail flank vorticity, generated downstream of the bump crest; again, no strong vortices are detected in this region,

(iii) centreline shear flow vorticity, only present on bumps which have a constant-height region between the flanks (for example, a wedge bump),

(iv) primary wake vortices; responsible for the measurable effects.

These are shown in Fig. 22 for a number of different bump shapes, the geometries of which were shown previously in Fig. 4d.

Although the question of generation mechanism remains unresolved, some progress is beginning to be made on understanding how to control the strength of the vortices. Recent work by Bruce et al. [7], studied a number of variants of the basic wedge geometry examined in previous work by the same author [6]. The results, shown in Fig. 23, indicate that the vortical structures are very similar for all three bumps, with marginally stronger downwash for the steepsided bump, in line with the idea that spanwise pressure gradients drive vortex production.

More convincing evidence for the role of spanwise pressure gradients was found by Colliss et al. [9], who correlated pressure gradients at different locations on a variety of bumps with the peak vorticity observed in the primary vortex struc- (a)

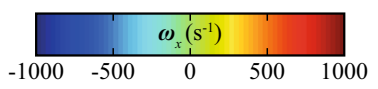

(b)

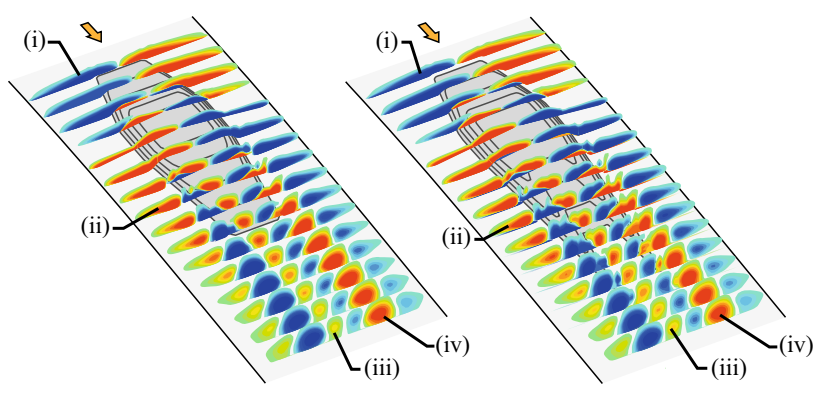

(c)

(d)

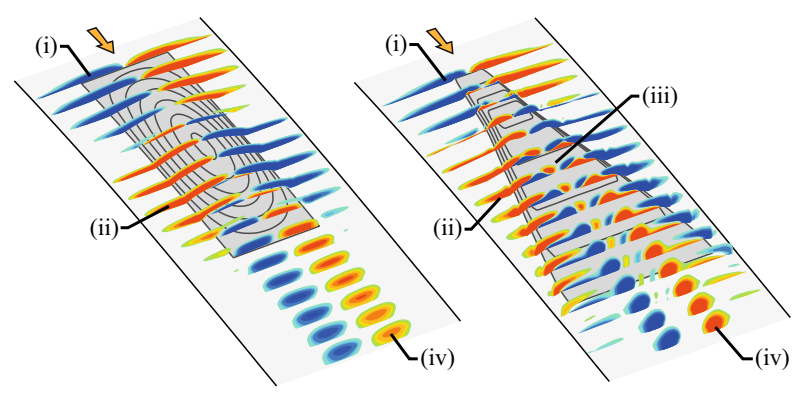

Fig. 22 Streamwise vorticity field determined from CFD for various bumps: a wedge bump (unpublished); b forked tail bump (unpublished); c hill-SCB, adapted from [9]; d extended tail bump, adapted from [9]

ture (identified in Fig. 22). Their results, shown in Fig. 24, suggest a strong link between spanwise pressure gradients on the ramp of a SCB and the vortex strength, with a weaker influence of spanwise pressure gradients on the tail and no trend at all with the streamwise pressure gradients.

The use of SCBs as a smart vortex generator is still a nascent subject, and as such further research is both required 


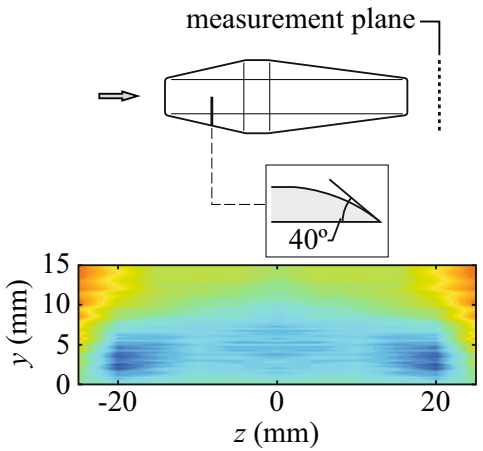

(a)

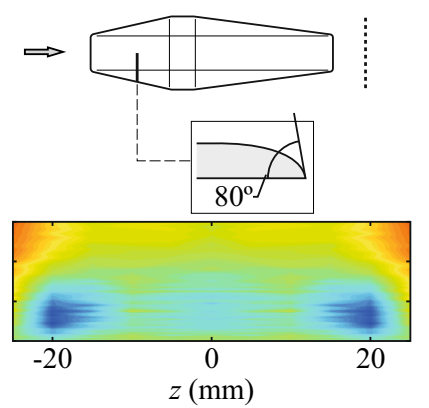

(b)

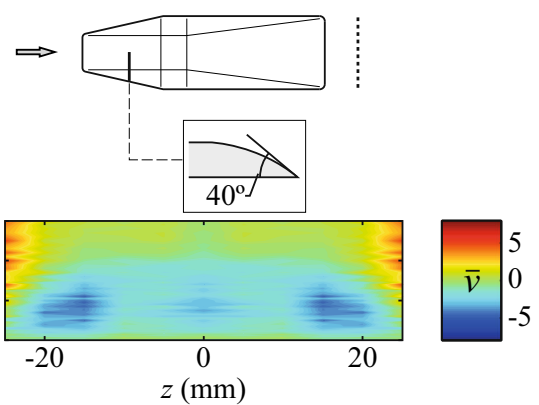

(c)

Fig. 23 Wall-normal velocities in a spanwise plane $4 \delta_{0}$ downstream of SCB tail, adapted from [7]: a standard wedge bump; b steep-sided bump; c wide-tailed bump. Velocity measurements were obtained using laser Doppler anemometry

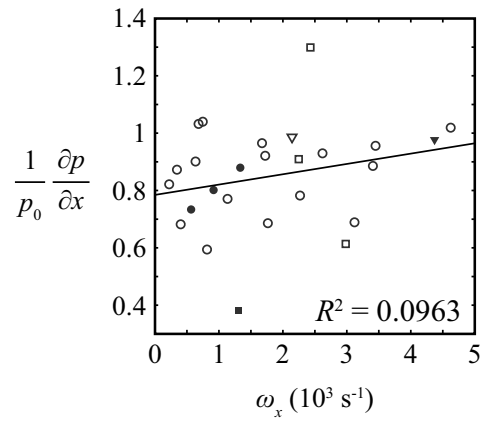

(a)

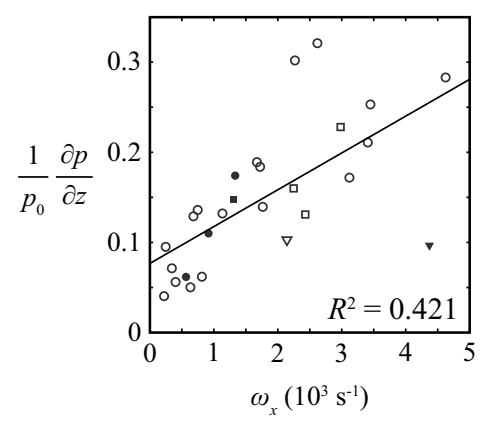

(b)

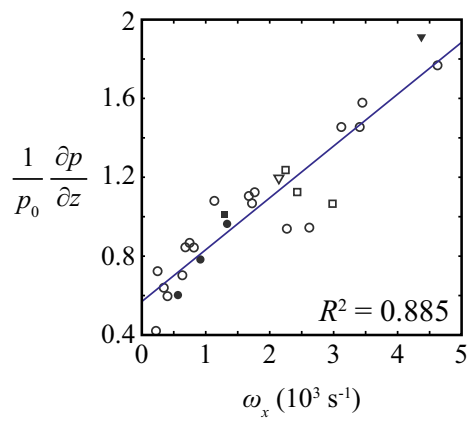

(c)

Fig. 24 Correlation of pressure gradients with vortex strength, from [9]: a streamwise pressure gradients on tail; $\mathbf{b}$ spanwise pressure gradients beneath primary vortex on tail; $\mathbf{c}$ maximum spanwise pressure gradients on ramp

and on-going. Although it appears that vortex strength may now be manipulated, this requires further testing, and controlling the trajectory and (not unrelated) decay of the vortices downstream requires further work.

\section{SCB performance}

In this section, we briefly summarise key conclusions from $\mathrm{SCB}$ research that relate to performance. We assess the potential of SCBs for drag reduction and transonic buffet alleviation, and also attempt to compare their performance potential with other flow control strategies.

\subsection{Drag reduction}

In terms of their potential for reducing drag, SCBs (both 2-D and 3-D) are most effective when applied to flows exhibiting strong and relatively stationary shock waves such as are found on laminar flow supercritical wings [33]. For the purpose of weakening such shock waves, our understanding of the potential and limitations of SCBs has reached a level of maturity where they can be considered a viable option as a laminar-flow enabling technology.

Consensus from the literature is that well-designed SCBs can offer significant improvements in aerodynamic performance (as quantified by the ratio $L / D$ ) at certain operating conditions when installed on conventional (turbulent) and natural laminar flow supercritical aerofoils or wings. Although reported performance benefits vary between different studies, maximum gains in $L / D$ of $10 \%$ or more have been widely reported. These reported gains are invariably achieved at relatively high values of $C_{L}$ and $M_{\infty}$, significantly in excess of typical transonic airliner cruise conditions (especially $C_{L}$, which often differs by a factor of 2 or more). Furthermore, the maximum values of $L / D$ achieved with SCBs at these high $C_{L} / M_{\infty}$ conditions often do not exceed the peak value of $L / D$ for the aerofoil without control at its nominal cruise conditions (where $C_{L}$ and $M_{\infty}$ are significantly lower). These observations are consistent with the premise that SCBs are only able to offer a performance benefit when wave drag reaches significant levels. However, only a small number of studies have actually attempted to quantify the contribution of wave drag towards overall drag $[4,19]$. Nonetheless, these studies do agree that SCBs are effective 
in reducing wave drag, and have a relatively small impact on other parameters (specifically viscous drag $\mathrm{C}_{D \text {,viscous }}$ ), at least on design.

Quantitative comparisons between the performance potential of 2-D and 3-D SCBs mounted on supercritical wings can be difficult to interpret. In almost all cases, a single 2-D $\mathrm{SCB}$ offers greater potential for improving $L / D$ at a single design point than an array of 3-D SCBs, while in terms of robustness, the opposite is true: arrays of 3-D SCBs do offer (albeit smaller) improvements in aerodynamic performance over a wider range of operating conditions than a single 2-D SCB [10]. Optimised 3-D SCBs tend to be taller than "equivalent' optimised 2-D SCBs, due to the decrease in 'effective turning angle' associated with 3-D devices [26]. SCB height is known to be an important parameter in determining the effectiveness of SCBs at different lift coefficients. It has been reported that taller SCBs are more effective at high lift coefficients, while lower SCBs are more effective in minimising drag at lower lift coefficients $[2,4,26]$. Optimising this apparent trade-off is an area of active research, and recent (currently unpublished) results from the NextWing program suggest that reducing the height of 3-D SCBs can significantly improve their robustness with only a small drop in peak $L / D$ reduction.

Finally, in selecting a suitable SCB for drag reduction, in addition to considering the desired design point $\mathrm{C}_{L}$ (which ultimately determines shock strength), the likely size of shock excursions from a mean position should be considered.

\subsection{Transonic buffet}

2-D SCBs: Birkemeyer et al. [4] proposed that a (2-D) SCB could postpone buffet onset to higher lift coefficients on a swept wing, based on observations of the impact of the SCB on the extent of shock-induced separation and the level of pressure fluctuations at the wing trailing edge. They used a combination of 2-D RANS computations and experiments to show that, at flow conditions approaching the buffet boundary, a SCB placed downstream of the main wing shock wave had a positive effect on these two criteria. However, similar benefits were not seen when the SCB was placed in the shock region (where it gave a beneficial reduction in drag). The authors reported that these results were broadly in agreement with observations first made by Ashill [2]: Essentially, 2-D SCBs may delay transonic buffet onset by introducing a region of attached flow between the shock wave and trailing edge of a supercritical wing, thus postponing complete flow breakdown (when separation extends from the shock to the trailing edge). Of course, they can only achieve this if they are placed between the shock wave and wing trailing edge, where they do not offer any drag benefit.
3-D SCBs: It has been demonstrated that the vortical flow structures produced by 3-D SCBs have the potential for exerting localised positive boundary layer control effects on the post-shock boundary layer in experiments and computations $[3,6,9,26,38]$. This effect has been likened to that of an array of vortex generators, which have been widely used to expand the performance envelopes of low speed wings and supercritical wings alike. This unique feature of 3-D SCBs offers the possibility of producing localised attached regions of flow in the region downstream of the shock location. For the case of a supercritical wing approaching buffet onset, an array of 3-D SCBs positioned close to the (streamwise) shock position can, in this way, prevent the occurrence of completely separated flow in the region of the shock and trailing edge, thus postponing flow breakdown to higher lift coefficients.

Eastwood and Jarrett [10] modelled the flow over an unswept transonic wing with various (2-D and 3-D) SCBs at a large range of different operating conditions (using a RANS code). They adopted a similar approach to that of Birkemeyer et al. [4] to assess the impact of SCBs on buffet onset; recording the onset and growth of flow separation (as determined by surface shear stress values) at both the shock location and the wing trailing edge. They used this information to determine the conditions at which these two regions of separation joined together to produce fully reversed flow from the shock to the trailing edge, which they took to be indicative of buffet onset, consistent with previous work $[11,28,36]$. Using this approach, they were able to explore the impact of SCBs on buffet characteristics at the same time as aerodynamic performance $L / D$. Two major conclusions from their study are as follows:

(i) 3-D SCBs show considerable potential as a means for postponing the onset of transonic buffet, as illustrated in Fig. 11 (shown earlier). Unlike previous studies with 2-D devices, 3-D SCBs are able to achieve this positive impact when they are installed close to the shock position on a supercritical wing.

(ii) Although 3-D SCBs can improve the buffet margin, they do so at a penalty to peak aerodynamic performance. This trade-off between $L / D$ and buffet boundary is well captured in Fig. 25.

Research that builds on the work of Eastwood and Jarrett is ongoing at IAG Stuttgart to characterise the buffet behaviour of an un-swept transonic wing with SCBs as part of the NextWing program [5]. A primary aim of this work is to establish design guidelines for bespoke SCBs that fulfil either a 'performance' or 'buffet alleviation' role. In addition, part of this work is focused on the assessment of different criteria for the analysis and quantification of buffet, including efforts to identify new criteria that relate relevant flow characteristics (such as flow separation area and vortex 


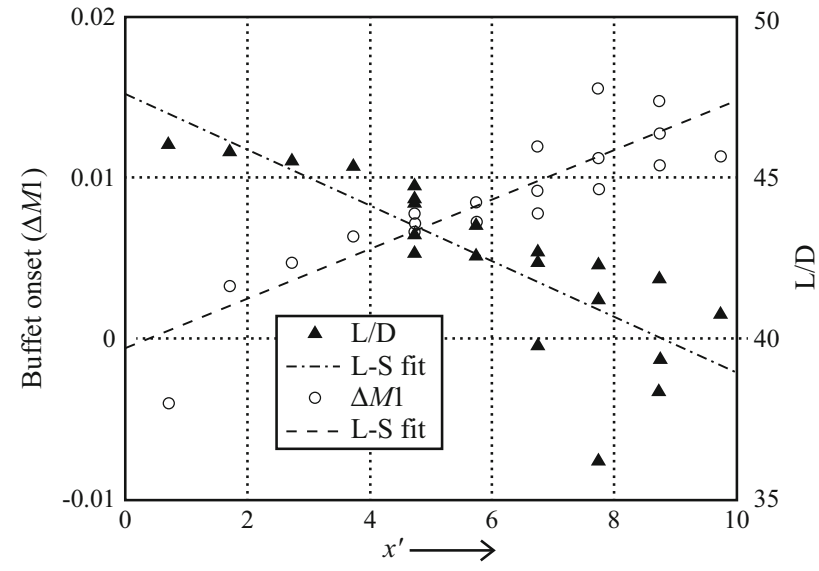

Fig. 25 Trade-off between aerodynamic performance $(L / D)$ and buffet alleviation $(\Delta M 1)$ for 3-D SCBs, from [10]. The variable $x^{\prime}$ denotes different test cases (SCB geometries and flow conditions), ordered in ascending peak vortex strength. Hence, data points on the left correspond to SCBs that produced low levels of vorticity and test cases to the right are SCBs that produced high levels of vorticity

strength) to buffet magnitude. The authors considered two different criteria for quantifying the onset of buffet and drew the following (preliminary) conclusions:

- RMS shock position $\neq 0$ : This criterion has significant flaws. Namely, it tends to over-predict the extent of the buffet regime, leading to conclusions which can be misleading.

- Deviation of $C_{L}-\alpha$ curve from linear: There is evidence that this approach, which is favoured by industry (Airbus), gives more reliable indications of buffet onset.

According to the second of these two criteria, a number of SCBs tested at IAG Stuttgart have shown promising potential for delaying the onset of buffet.

\subsection{Comparisons with other supersonic flow control strategies}

Numerous review papers and research projects have made comparisons between SCBs and other (supersonic and transonic) flow control strategies $[1,3,33]$. A common conclusion amongst these studies and reviews is that 3-D SCBs offer a unique combination of promising performance potential (in terms of drag and/or buffet) and practicality (in terms of ease of installation, cost, weight, etc.) for supercritical wings. The concept of targeting wave drag using so-called 'shock control' is not something that is unique to SCBs. The basic principle of shock control is well summarised by Ogawa and Babinsky [23], who explain that any flow control device that causes a flow deflection has the potential to reduce wave drag. However, although other (passive) shock-control techniques have been considered (such as the perforated surface cavity), it has been found that such techniques invariably incur prohibitively high levels of viscous drag which outweigh any wave drag benefit $[1,33]$.

In recent years, as our knowledge of the (vortical) flow structures associated with 3-D SCBs has matured, researchers have begun to compare these devices with vortex generators. In one such study, Colliss et al. [9] found that the strength of the vortices produced by a 3-D SCB is of the same order of magnitude as those of vortex generators (as reported by Ashill et al. [1]), when expressed in terms of the non-dimensional circulation parameter $\Gamma /\left(u_{\tau} h\right)$ (where $u_{\tau}$ is the local uncontrolled friction velocity and $h$ is the SCB or vortex generator height). However, Colliss et al. [9] also explain that quantifying the 'strength' of the vortices is not always trivial: as the existence of strong shear flows, in which the vortices are embedded, can make it difficult to extract the contribution to $\Gamma$ of the vortex itself. Thus it is difficult to compare SCBs to vortex generators in terms of their peak vorticity. Work is ongoing at Imperial College London and Cambridge University to explore the importance of parameters such as peak vorticity and total circulation to better characterise the vorticity produced by 3-D SCBs.

\section{Directions for future research and development}

The primary SCB application recognised in literature is as a means of reducing the wave drag of laminar flow wing configurations [33], hence acting as a potential enabling technology for realising a practical laminar flow wing. In this context, our understanding of (2-D and 3-D) devices has reached a level of maturity where further studies aimed purely at quantifying their drag-saving potential for quasi-2D wing arrangements is of limited value. Future research should focus on the wider challenges relevant to the integration of SCBs within real wing designs.

This section is split into two parts: First, we consider aspects of SCB behaviour where our current understanding is lacking; second, we briefly review two alternate concepts and applications for SCBs.

\subsection{Challenges in understanding}

\subsubsection{Impact of unsteadiness on SCB performance}

SCB performance in unsteady flow is not well understood. Although some quasi-unsteady observations have been made (such as the stabilising effect of a wedge-shaped SCB), the transient flow produced by a SCB in response to rapid changes in flow conditions has not been studied. This is of concern given that considerable interest in SCBs is focused on their potential to control the onset of buffet; an inher- 
ently unsteady phenomenon. Although the underlying mechanisms of how SCBs favourably influence buffet onset are thought to be understood in broad terms (breaking up large regions of separation) no unsteady experiments or simulations exist that verify this. For example, the results of Eastwood and Jarrett [10], although convincing, were obtained with RANS computations.

In this respect, further wind tunnel tests are essential. This is because the flow on a supercritical wing at near-buffet conditions is especially challenging for practical existing CFD (RANS), which struggles to cope up with the effects of compressibility and unsteadiness in flows with potentially significant levels of separation. Although more high-fidelity computational approaches (DNS/LES/hybrid methods) offer an improved capability for predicting such flows; the high Reynolds numbers associated with real flight conditions (and in fact most scale model wind tunnel tests) are far out-ofreach of these tools and are likely to remain so for at least the next 1-2 decades.

\subsubsection{SCBs on real 3-D swept wings}

Since the practical goal of SCB research is to incorporate the technology into an aeroplane wing, an important area where progress must be made is swept wing flows and their effects on the SCB physics. A real finite-span wing flow is quite different from the unswept flows used in most SCB investigations to date, and bears only local similarities to the infinite-span swept flows tested in some studies, such as that of Birkemeyer et al. [4]. Currently, no investigation of the SCBs' effect on the more complicated and three-dimensional flows at the wing root and tip have been performed; an understanding of how best to approach these situations is therefore needed. In any case, the question of how best to design a swept SCB remains: bending the SCB planform to follow surface streamlines; using an unswept SCB profile; and creating asymmetric SCB flanks are different methods tested so far, with no one appearing better than the others.

Another important consideration is the direct boundary layer control exerted by the SCB: if it is to be used a smart vortex generator, then for a swept wing it must produce corotating vortices. So far, every SCB tested has produce a counter-rotating vortical wake. However, it may be possible to suppress one of the vortices by reducing the spanwise pressure gradients on one side of the SCB ramp. Indeed, some (currently unpublished) preliminary work at the University of Stuttgart apparently shows exactly this effect.

A particularly useful tool for the above investigations will be the development of a simple experimental set-up which allows a canonical model of a swept flow to be generated, much in the same way as has been done for the unswept case. With this, a joint experimental and numerical study can set about examining the flow in detail, aiming to answer the questions using physical insight to guide and aid the interpretations of eventual optimisation exercises.

\subsection{Future concepts for SCB research}

\subsubsection{Active (morphing) SCBs for wings}

The sensitivity of SCB performance to external flow conditions has led to interest in the potential of active (adaptive or morphing) devices $[22,32]$. The potential to deploy one or more SCBs in favourable flow conditions (where they can give an improvement in $L / D$ or delay buffet onset) and remove them at off-design conditions (where an SCB would incur a performance penalty relative to the clean wing) is clearly desirable. However, as with many active flow control concepts, the idea has often been dismissed over concerns about the required complexity and added weight of such a system, thereby reducing the potential advantages of (particularly 3-D) SCBs over other flow control technologies in terms of simplicity, weight, and ease of retrofit.

Despite these potential pitfalls, results from a recent study at Imperial College London by Rhodes and Santer [30,31] suggest that morphing SCBs may be a viable possibility after all, thanks to recent advances in materials and actuation technology and also the adoption of a new (multidisciplinary) approach to SCB design [30]. This new approach involves considering structural and material limitations as well as aerodynamic performance to generate SCB designs that offer a compromise between simplicity, low actuation forces and material stresses as well as good aerodynamic performance.

Rhodes and Santer studied the deployment of a wedgetype 3-D SCB from an initially flat plate as well as a 2-D SCB. They concluded that morphing wedge-shaped 3D SCBs present significant challenges. This is primarily because sharp corners require large changes in Gaussian curvature over small regions which require membrane compliance above the limits of many current material technologies. Ongoing work is looking at more gently curved geometries such as the Hill-SCB and 2-D optimal designs.

\subsubsection{SCBs in intakes}

Whilst the majority of the work on SCBs has focussed on their application to transonic aerofoils, a recurring theme has been the SCBs' ability to exert a level of boundary layer control through the production of streamwise vortices. This, coupled with the obvious shock control benefits, has lead to the suggestion that the SCB may have an alternative application in controlling the flow in a supersonic engine intake [3]. In this role the SCB would be required to modify the shock/boundary layer interaction such that the downstream flow is more uniform and carries a higher total pressure recovery to the compressor entry than the uncontrolled flow. 
Some numerical studies of using SCBs in an intake have already been conducted by Kim and Song [14-16], who showed that it is possible to optimise a three-dimensional bump which improves the performance of a mixed compression intake. However, further insight into the flow physics and a more intake-neutral analysis is required to assess the potential of SCBs in this regard more fully. One useful way in which this might proceed is to follow the approach of Titchener et al. [37], who defined a simplified canonical intake flow in a blowdown supersonic wind tunnel and used it to study various configurations of vortex generators. The simplest metric of performance in these tests would be the degree to which the bumps can suppress (or reduce) boundary layer separation. This could be measured either by direct means (surface oil flow visualisation) or via surface pressure distributions. Additionally, following [37], the total pressure downstream at the simulated aerodynamic interface plane (AIP) could serve as a measure of the flow distortion in the viscous region.

It is apparent from the above discussion that the requirements of a SCB in an intake are not too dissimilar from those for the control of transonic buffet on an aerofoil. Thus it may be that the design of SCBs suited to either application turns out to be similar in their general characteristics—namely geometry, vortex production capability and placement on the wing or in the intake. Of course, this is all the subject to further research in the area.

Acknowledgments The authors are grateful to the contributors at a "Transonic flow control technologies workshop" held at Imperial College London in June 2012. Much of the material discussed at this workshop is included in the papers referenced in this article. The NextWing programme described as part of this work has received funding from the European Union's Seventh Framework Programme (FP7/2007-2013) for the Clean Sky Joint Technology Initiative under grant agreement no. 271843.

Open Access This article is distributed under the terms of the Creative Commons Attribution License which permits any use, distribution, and reproduction in any medium, provided the original author(s) and the source are credited.

\section{References}

1. Ashill, P.R., Fulker, J.L., Hackett, K.C.: A review of recent developments in flow control. Aeronaut. J. 109(1095), 205-232 (2005)

2. Ashill, P.R., Fulker, J.L., Shires, A.: A novel technique for controlling shock strength of laminar-flow aerofoil sections. DGLR Bericht Part 6(92-01-022), 175-183 (1992)

3. Babinsky, H., Ogawa, H.: SBLI control for wings and inlets. Shock Waves 18, 89-96 (2008)

4. Birkenmeyer, Rosemann, H.J., Stanewsky, E.: Shock control on a swept wing. Aerospace Sci. Technol. 4, 147-156 (2000)

5. Bogdanski, S., Nübler, K., Lutz, T., Kramer, E.: Numerical investigation of the influence of shock control bumps on the buffet characteristics of transonic airfoil. In: 18th DGLR-Symposium of STAB, Stuttgart, Germany, November 6-7. ISSW (2012)
6. Bruce, P.J.K., Babinsky, H.: Experimental study into the flow physics of three-dimensional shock control bumps. J. Aircraft 49(5), 1222-1233 (2012)

7. Bruce, P.J.K., Colliss, S.P., Babinsky, H.: Three-dimensional shock control bumps: effects of geometry. In: 52nd AIAA Aerospace Sciences Meeting and Exhibit, Maryland, WS. AIAA paper 20140943 (January 2014)

8. Colliss, S.P., Babinsky, H., Nübler, K., Lutz, T.: Joint experimental and numerical approach to three-dimensional shock control bump research. AIAA J. 52(2), 436-446 (2014)

9. Colliss, S.P., Babinsky, H., Nübler, K., Lutz, T.: Vortical structures on three-dimensional shock control bumps. In: 51st AIAA Aerospace Sciences Meeting and Exhibit, January 7-10, Grapevine, TX. AIAA paper 2013-0403 (January 2013)

10. Eastwood, J.P., Jarrett, J.P.: Toward designing with threedimensional bumps for lift/drag improvement and buffet alleviation. AIAA J. 50(12), 2882-2898 (2012)

11. ESDU. An introduction to aircraft buffet and buffeting, ESDU TR 87012. Technical report

12. Holden, H.A., Babinsky, H.: Shock/boundary layer interaction control using 3d devices. In: 41st AIAA Aerospace Sciences Meeting and Exhibit, January 6-9, Reno, NV. AIAA paper 2003-0447, blackJanuary (2003)

13. Milholen, II W.E., Owens, L.R.: On the application of contour bumps for transonic drag reduction. In: 43rd AIAA Aerospace Sciences Meeting and Exhibit, Reno, NV. AIAA (January 2005)

14. Kim, S.D.: Aerodynamic design of a supersonic inlet with a parametric bump. AIAA J. Aircraft 46(1), 198-202 (2009)

15. Kim, S.D., Song, D.J.: A numerical analysis of 3D flow field in supersonic bump-type inlet. J. Mech. Sci. Technol. 21(2), 327-335 (2007)

16. Kim, S.D., Song, D.J.: Numerical study of the performance of supersonic inlets with various three-dimensional bumps. J. Mech. Sci. Technol. 22, 1640-1647 (2008)

17. König, B., Patzold, M., Lutz, T., Kramer, E., Rosemann, H., Richter, K., Uhlemann, H.: Numerical and experimental validation of threedimensional shock control bumps. AIAA J. Aircraft 46(2), 675682 (2009)

18. Lee, B.H.K.: Self-sustained shock oscillations on airfoils at transonic speeds. Progress Aerosp. Sci. 37, 147-196 (2001)

19. Lee, D.S., Periaux, J., Onate, E., Gonzalez, L.F., Qin, N.: Active transonic aerofoil design optimization using robust multiobjective evolutionary algorithms. AIAA J. Aircraft 48(3), 1084-1094 (2011)

20. McGowan, A.R.: AVST morphing project research summaries in fiscal year 2001, nasa tm-2002-2 11769. Technical report, NASA (August 2002)

21. McIntosh, S.C., Qin, N.: The influence of transition onset location on the performance of shock control bumps. Aeronaut. J. 117(1196), 1137-1151 (2013)

22. Monner, H., Breitbach, E., Bein, T., Hanselka, H.: Design aspects of the adaptive wing - the elastic trailing edge and the local spoiler bump. Aeronaut. J. 104(1032), 89-95 (2000)

23. Ogawa, H., Babinsky, H.: Evaluation of wave drag reduction by flow control. Aerosp. Sci. Technol. 10(1), 1-8 (2006)

24. Ogawa, H., Babinsky, H.: Experimental investigation of 3D shock/boundary layer interaction control in transonic flow. In: 44th AIAA Aerospace Sciences Meeting and Exhibit, January 9-12, Reno, NV. AIAA paper 2006-0879 (January 2006)

25. Ogawa, H., Babinsky, H.: Wind-tunnel setup for investigations of normal shock wave/boundary-layer interaction control. AIAA J. 44(11), 2803-2805 (2006)

26. Ogawa, H., Babinsky, H., Pätzold, M., Lutz, T.: Shockwave/boundary-layer interaction control using three-dimensional bumps for transonic wing. AIAA J. 46(6), 1442-1452 (2008) 
27. Pätzold, M., Lutz, T., Kramer, E., Wagner, S.: Numerical optimization of finite shock control bumps. In: 44th AIAA Aerospace Sciences Meeting and Exhibit, January 9-12, Reno, NV. AIAA paper 2006-1054 (January 2006)

28. Pearcey, H.: A method for the prediction of the onset of buffeting and other separation effects from wind tunnel tests on rigid models, agard report 223. Technical report, AGARD (1958)

29. Qin, N., Wong, W.S., LeMoigne, A.: Three-dimensional contour bumps for transonic wing drag reduction. IMechE Part G 222(5), 619-629 (2008)

30. Rhodes, O., Santer, M.: Structural optimization of a morphing shock control bump. In: 52nd AIAA/ASME/ASCE/AHS/ASC Structures, Structural Dynamics and Materials Conference, Denver, Colorado. AIAA Paper 2011-2129 (April 4-7 2011)

31. Rhodes, O., Santer, M.: Aeroelastic optimization of a morphing 2d shock control bump. In: 53rd AIAA/ASME/ASCE /AHS/ASC Structures, Structural Dynamics and Materials Conference, Honolulu, Hawaii. AIAA Paper 2012-1577 (April 23-26 2012)

32. Stanewsky, E.: Aerodynamic benefits of adaptive wing technology. Aerosp. Sci. Technol. 4, 439-452 (2000)
33. Stanewsky, E., Délery, J., Fulker, J., de Matteis, P., editors. Synopsis of the project Euroshock II, Notes on Numerical Fluid Mechanics and Multidisciplinary Design: Drag Reduction by Shock and Boundary Layer Control-Results of the Project EUROSHOCK II, Vol. 80. Springer, Berlin (2002)

34. Tai, T.C.: Theoretical aspects of dromedaryfoil, report 77-0104. Technical report, David W. Taylor Naval Ship Research and Development Center (November 1997)

35. Tai, T.C., Huson, G.G., Hicks, R.M., Gregorek, G.M.: Transonic characteristics of a humped airfoil. AIAA Paper 87-1239 (June 1987)

36. Thomas, F., Redeker, G.: Facilities and Techniques for Aerodynamic Testing at Transonic Speeds and High Reynolds, AGARD CP-83. Technical Report, Goettingen, Germany (1971)

37. Titchener, N.A., Babinsky, H.: Shock wave/boundary-layer interaction control using a combination of vortex generators and bleed. AIAA J. 51(5), 1221-1233 (2013)

38. Wong, W.S., Qin, N., Sellars, N., Holden, H., Babinsky, H.: A combined experimental and numerical study of flow structures over three-dimensional shock control bumps. J. Aerosp. Sci. Technol. 12, 436-447 (2008) 